\title{
Sulfated host glycan recognition by carbohydrate sulfatases of the human gut microbiota
}

*1,2Ana Luis, ${ }^{3}$ Arnaud Baslé, ${ }^{4}$ Dominic P Byrne, ${ }^{4}$ Gareth SA Wright, ${ }^{4}$ James London, ${ }^{2}$ Jin Chunsheng, ${ }^{2}$ Niclas Karlsson, ${ }^{2}$ Gunnar Hansson, ${ }^{4}$ Patrick Eyers, ${ }^{5}$ Mirjam, Czjzek, ${ }^{5}$ Tristan Barbeyron, ${ }^{4}$ Edwin A Yates, ${ }^{1}$ Eric C. Martens, and ${ }^{* 4}$ Alan Cartmell

${ }^{1}$ Department of Microbiology and Immunology, University of Michigan, Ann Arbor, MI 48109, USA

${ }^{2}$ Department of Medical Biochemistry, University of Gothenburg, Box 440, 40530 Gothenburg, Sweden

${ }^{3}$ Institute for Cell and Molecular Biosciences, Newcastle University, Newcastle upon Tyne, United Kingdom

${ }^{4}$ Department of Biochemistry and Systems Biology, Institute of Systems, Molecular and Integrative Biology, University of Liverpool, Liverpool L69 3BX, United Kingdom ${ }^{5}$ Sorbonne Université, Univ Paris 06, CNRS, UMR 8227, Integrative Biology of Marine Models, Station Biologique de Roscoff, CS 90074, Roscoff, Bretagne, France.

*Correspondence to:

Alan.Cartmell@liverpool.ac.uk

ana.luis@medkem.gu.se

\section{Abstract}

The vast microbial community that resides in the human colon, termed the human gut microbiota, performs important roles in maintaining host health. Sulfated host glycans comprise both a major nutrient source and important colonisation factors for this community. Carbohydrate sulfatases remove sulfate groups from glycans and are essential in many bacteria for the utilisation of sulfated host glycans. Additionally, carbohydrate sulfatases are also implicated in numerous host diseases, but remain some of the most understudied carbohydrate active enzymes to date, especially at the structural and molecular level. In this work, we analyse 7 carbohydrate sulfatases, spanning 4 subfamilies, from the human gut symbiont Bacteroides thetaiotaomicron, a major utiliser of sulfated host glycans, correlating structural and functional data with phylogenetic and environmental analyses. Together, these data begin to fill the 
knowledge gaps in how carbohydrate sulfatases orchestrate sulfated glycan metabolism within their environment.

\section{Introduction}

The human gut microbiota (HGM) is a vast microbial community ${ }^{1}$ that makes important contributions to its host's physiology by contributing to an array of biological processes, including protection from pathogens ${ }^{2}$, regulating the immune system ${ }^{3}$, and providing up to $10 \%$ of the hosts energy needs through complex carbohydrate fermentation ${ }^{4}$. The Bacteroidetes constitute a major phylum within this community. These bacteria thrive within this competitive environment by metabolising complex glycans derived from both the diet, namely plant cell wall polysaccharides and other dietary fibers ${ }^{5-7}$, and the host, which continuously secretes glycan-rich substances such as mucins found in the protective mucus layers of the body ${ }^{8-10}$. Some Bacteroides dedicate as much as $20 \%$ of their genome to glycan metabolism ${ }^{11,12}$ and members of this phylum arrange their CArbohydrate Active enZymes (CAZymes) into polysaccharide utilisation loci (PUL) ${ }^{13}$, which are sets of genes that are genetically colocalised and co-regulated in response to a particular glycan.

The ability to metabolise sulfated host glycans such as glycosaminoglycans (GAGs), which include heparan sulfate $(\mathrm{HS})^{8}$ and chondroitin sulfate $(\mathrm{CS})^{9}$, and colonic mucin O-glycans ${ }^{10}$ (Figure 1) has been shown for several Bacteroides species. These complex carbohydrates are also important colonisation factors ${ }^{14,15}$, and GAGs have also been shown to be high priority nutrients ${ }^{16,17}$. Thus, the effects of host glycans on the microbiota composition could be profound. Carbohydrate sulfatases are enzymes that catalyse the de-sulfation of host glycans and are essential for their utilisation ${ }^{8}$ by bacteroides species of the HGM. Additionally, carbohydrate sulfatases produced by gut microbes have been implicated in inflammatory bowel disease (IBD) in humans ${ }^{18,19}$, and directly linked to promoting colitis in a susceptible mouse mode ${ }^{20,21}$. A recent study has also demonstrated that carbohydrate sulfatase activity is a keystone step in colonic mucin metabolism and their loss results in an inability of at least one Bacteroides species to utilise colonic mucin and effectively colonise the mouse gut ${ }^{10}$. The model organism Bacteroides thetaiotaomicron ( $B$. theta) possesses at least 28 sulfatases spread amongst several PULs of both known, and unknown, function in glycan degradation ${ }^{8-10}$. Despite the important role of carbohydrate sulfatases in glycan degradation, particularly host glycans, there is a 
significant knowledge gap regarding the structural basis behind the substrate recognition by these enzymes.

Sulfatases are catalogued in the SulfAtlas database (http:/labims.sbroscoff.fr/sulfatlas $/)^{22}$. These enzymes are currently divided into four families, S1-S4, based on sequence similarity, fold, and catalytic mechanism. The $\mathrm{S} 1$ family is the largest family comprising $\sim 90 \%$ of all sulfatase sequences, is found throughout all domains of life, and is currently the only family that has been significantly implicated in carbohydrate metabolism. There are currently 72 S1 subfamilies (denoted as S1_X) comprising a total of 36,815 individual sulfatases yet less than $1 \%$ have had their activities analysed and only $\sim 10$ unique carbohydrate sulfatase structures exist. This makes $\mathrm{S} 1$ carbohydrate sulfatases some of the most poorly characterised CAZymes to date.

The $\mathrm{S} 1$ family is part of the alkaline-phosphatase-like superfamily. All S1 sulfatases require either a Cys or Ser residue, within the core consensus sequence C/S-X-P/A-S/X-R, to be co-transitionally transformed into formylglycine (FGly) to be catalytically active ${ }^{23}$. Calcium is an essential cofactor for all $\mathrm{S} 1$ sulfatases, whilst the catalytic acid is likely an invariant His or Lys that coordinates with the scissile sulfoester linkage. The subsite nomenclature for carbohydrate sulfatases is such that the invariant sulfate binding site is denoted the $S$ site. The $S$ site sulfate is appended to the 0 subsite sugar. Subsites then increase in number (i.e. $+1,+2,+3$ ) as the sugar moves toward the reducing end (free 01 ) and decreases in number as the sugar chain moves towards the non-reducing end (i.e. $-1,-2,-3)^{24}$.

Here, we describe the structures of 7 Bacteroides $\$ 1$ carbohydrate sulfatases, 6 in complex with ligand (Figure S1), along with detailed biochemical, mutagenic, and bioinformatic analyses. The sulfatases span 4 different subfamilies, with three of the structures representing the first structural description of S1_16 and S1_46 subfamilies, whilst the additional structures of S1_11 and S1_15 subfamilies reveal how nonconserved areas of the proteins are adapted to increase specificity for the particular glycan targeted by the PUL in which they reside. The data reveal the exquisite specificity that $\mathrm{S} 1$ carbohydrate sulfatases utilise to recognise their cognate sulfated glycan within the human colon and lay the foundations for developing strategies to manipulate this specificity to improve microbiome health, and thus that of the host. 
102

103

104

105

106

107

108

109

110

111

112

113

114

115

116

117

118

119

120

121

122

123

124

125

126

127

128

129

130

131

132

133

134

\section{Results}

\section{Conserved features of S1 formylglycine sulfatases}

Consistent with the previously characterized structures, all the S1 sulfatases investigated here adopt an $\alpha / \beta / \alpha$ fold for the core $N$-terminal domain which is abutted by a smaller, C-terminal, 'sub domain' (Figure 2a). The sulfate binding site (S site) is invariant across the $S 1$ family. The FGly residue sits at the base of the pocket in the consensus sequence $\mathbf{C} / \mathbf{S}-\mathrm{X}-\mathrm{P} / \mathrm{A}-\mathrm{S} / \mathrm{X}-\mathrm{R}$. The varying ratios of $\mathrm{Cys} / \mathrm{Ser}$ at the critical FG position are 83/17 for S1_11, 82/18 for S1_15, 85/15 for S1_16 and 84/15 for S1_46 (Figure 2b). Pro is conserved in $95 \%$ of the sequences except in S1_46 where Pro exists $90 \%$ of the time with Ala being the other $10 \%$ (Figure 2b). Although $B$. theta sulfatases exclusively possess Ser, the favouring of Cys over Ser reflects that only organisms inhabiting anaerobic environments are currently known to convert Ser to FGly ${ }^{23}$. Individual enzymes are subsequently referred by their gene/locus tag number with the corresponding activity in superscript (e.g., BT19183S-GIcNAc). All structures solved were Ser variants and, with exception of BT1918 3 S-GICNAc, had occupation of calcium in the metal binding site. In the S1_11, S1_15, and S1_16 subfamilies three Asp residues, a GIn/Asn and the FGly coordinate the calcium with an octahedral coordination completed by the incoming sulfate substrate (Figure 2c). In BT1918 3 S-GICNAC one of the conserved Asp is replaced by Q292 and GIn/Asn by H318 (Figure 2c). Q292 is positioned further away than the conserved Asp and may change the coordination to trigonal bi-pyramid and a weaker calcium binding capacity. Additionally, the absence of the FGly residue will also make calcium binding weaker in the BT1918 ${ }^{3 S-G I C N A C}$ structure. This was previously observed in BT1596 $2 \mathrm{~S}-\triangle 4,5 \mathrm{UA}$, an S1_9 sulfatase, where both Asp and GIn/Asn residues are replaced by $\mathrm{His}^{8}$ (Figure

\section{2c)}

\section{S1_46 sulfatases target rare linkages found in host glycosaminoglycans}

BT1918 ${ }^{3 S-G I C N A C}$ is a dimer in solution (Figure S2a) and the only sulfatase identified to date that cleaves sulfate from the 03 position of $3 S, 6 S-D-N$-acetylglucosamine $(3 S, 6 S-G I c N A c)^{10}$, a known component of the host glycans heparin (Hep) and heparan sulfate (HS) (Figure 1). The $O 6$ sulfate does not appear to contribute to substrate specificity, and mutation of W173 to Ala, the only residue located near the O6 sulfate, causes no significant loss in activity (Figure $\mathbf{3 a}, \mathbf{3 b}, \mathbf{S 2} \mathbf{b}$ and Table S1). Out of the 
five residues in the carbohydrate binding region three interact with the $N$-acetyl group (Y94, R327, and Y408) (Figure 3a). Mutation of Y94, Y408, and R327 individually to Ala causes $\mathbf{a} \sim 40, \sim 20$, and $\sim 10$-fold loss in activity, respectively (Figure $\mathbf{3 b} \mathbf{b}, \mathbf{S} \mathbf{b}$, S2c, and Table S1). This suggests that the interactions with $N$-acetyl group are a major specificity determinant in S1_46 sulfatases. A finding that is consistent with the lack of activity of BT1918 3 S-GICNAc on 3S- and 3S,6S-glucosamine ${ }^{10}$ (Figure S2d). The sulfatase activity was not affected by the mutation of Y94 and Y408 to Phe indicating their action is through the steric/stacking contributions of the phenol ring. N174 binds to the endocyclic ring oxygen via $\mathrm{N} \delta 2$ and $\mathrm{R} 143$ coordinates with $\mathrm{O} 4$ via $\mathrm{N} \eta 1$, both helping to orientate the $\mathrm{O} 3$ of the sugar (Figure 3a). The mutation of N174 and R143 to Ala causes a $\sim 30$ and $\sim 40$ fold reduction in catalytic activity, respectively (Figure $3 b$ and Table S1), indicating that these residues have a significant role in the substrate recognition. Phylogenetic analyses show that N174 and R143 are conserved in $87 \%$ and $76 \%$, respectively, of the aligned S1_46 sequences. Additionally, Y94 and R327 are conserved in $\sim 65 \%$ sequences. Y94 is present in the motif GRVGYGDE and is replaced by Asn and Phe $18 \%$ and $10 \%$ of the time, respectively (Figure 3c). Mutational data indicate that the Phe substitution does not affect activity. It is of note that $\mathrm{Y} 94, \mathrm{~N} 174, \mathrm{R} 327$, and $\mathrm{Y} 408$ are invariant within Bacteroidetes inhabiting the human gastrointestinal tract (Table S2). In fact, the majority of sequences from S1_46 are derived from human sources and include several human pathogens, including Clostridium difficile $P 28$, a sequence which contains residues equivalent to $Y 94, N 174$, and R327 but not Y408 (Table S2). Interestingly, Y408 is only found in 26\% of S1_46 sequences, within a HATCY motif, but is present in all Gram-negative bacteria within this subfamily indicating some phylum level differences.

\section{Aromatic stacking is essential for S1_16 4S-Gal/GalNAc sulfatases of the HGM} BT30574S-Gal/GalNAc and BT37964S-Gal/GalNAc are both 4S-D-galactose/Nacetylgalactosamine (4S-Gal/GalNAc) sulfatases, displaying a mix of monomeric and dimeric species, likely a consequence of heterologous expression, (Figure S3 and Figure S4a,b,c), and appear to bind Gal/GalNAc in similar manners (Figure 4a,b). In the 0 subsite of both sulfatases a critical aromatic residue, W109, stacks against the $\alpha$ face of the sugar ring whilst $\mathrm{O} 3$ is coordinated via $\mathrm{N} \varepsilon 2$ of $\mathrm{H} 423$, in BT30574S-Gal/GalNAc, and the indole nitrogen of W431, in BT37964S-Gal/GalNAc, (Figure 4a, b). In both 
enzymes mutation of W109 to Ala caused loss of any quantifiable activity but some trace activity was observed qualitatively, after extended incubations (Table S1 and Figure S4d,e). The mutation of the residue coordinating $O 3$ causes $\sim 10$ and $\sim 50$ fold reduction in activity for BT30574S-Gal/GalNAc and BT3796 4 S-Gal/GalNAc, respectively (Table S1 and Figure S4d,e). In BT30574S-Gal/GalNAc an additional interaction is made via N $\delta 1$ of $\mathrm{H} 182$ to $\mathrm{O} 6$ and mutation to Ala causes $\sim 20$ fold reduction in activity (Figure $4 \mathrm{a}$ and Table S1). In BT37964S-Gal/GalNAc a glycine resides in place of $\mathrm{H} 182$ but a Trp residue, W332, lies opposite the position (Figure 4b). It is possible that both H182 (BT305745Gal/GalNAc) and W332 (BT37964S-Gal/GalNAc) residues could also contribute to the binding of a +1 sugar, through a stacking interaction, should such a substrate encounter the enzymes (Figure 4a, b). Interestingly, despite its critical nature, W109 is only observed in $37 \%$ of analysed S1_16 sequences. However, this residue is found in 84 $\%$ of the sequences from organisms residing in the human gut, but drops to $17 \%$ for sequences from a marine or aquatic environments where Val is most frequently observed (Figure 4c and Table S3). The aromatics Phe and Tyr, replace W109 at a frequency of $\sim 8 \%$ and $\sim 6 \%$, respectively, and could theoretically perform the same stacking role as W109 (Figure 4c and Table S3). Bioinformatic analysis shows that $72 \%$ of the total S1_16 sequences were from a marine or aquatic environment with terrestrial and human sources making up most of the remainder in roughly equal proportion (Table S3). These data suggest that only the subset of S1_16 sulfatases possessing an equivalent residue to W109 will be active as 4S-Gal/GalNAc sulfatases and that this activity is mostly restricted to human gut bacteria; $\mathrm{S} 1$ _16 sulfatases from marine environments likely target a different, but potentially somewhat analogous, sulfated linkage.

\section{Defining the conserved and variable features driving 6S-Gal/GalNAc recognition}

The 4 S1_15 enzymes characterised here are all monomeric (Figure S5a) and exclusively target 6 S-D-galacto configured substrates ${ }^{10}$ through a conserved mechanism that was observed previously for BT33336S-GalNAc, a sulfatase that specifically cleaves $6 \mathrm{~S}-$ GalNAc $^{9}$. These conserved recognition features are D176, R177 and H227 in BT46316S-Gal/GalNAc, D170, R171, and H220 in BT1624 ${ }^{6 S-G a l / G a l N A c}$, and D162, R163 and H212 in BT31096S-Gal (Figure 5a, b). In all three enzymes the His residue co-ordinates with $\mathrm{O} 3$ via $\mathrm{N} \varepsilon 2$, whilst Asp and Arg hydrogen bond to 04 
through $\mathrm{O} \delta 2$ and $\mathrm{N} \eta 1$, respectively (Figure $5 \mathrm{a}$ ). The His residue resides in a small motif (HDQSIV) present in $56 \%$ of all sequences but, within the motif, the His exists at a $99 \%$ frequency. The Asp and Arg are found within the conserved motif FIMAATGDRVP but Asp and Arg are only found at frequencies of $74 \%$ and $56 \%$, respectively (Extended data 1 and Table S4). Overall this means the 'recognition triad' is conserved in $56 \%$ (523) of all sequences analysed, suggesting that at least half of the members of this subfamily target galacto configured carbohydrates. Analysis of the environment from which the S1_15 sequences were derived reveal that $\sim 65 \%$ of all S1_15 sequences are from a marine environment and that nearly 70 $\%$ of these lack the galactose recognition triad (Table S4). By contrast, $83 \%, 80 \%$ and $95 \%$ of sequences from human, animal, and terrestrial sources, respectively, contain the galactose recognition triad (Table S4). This suggests that S1_15 subfamily members target at least two types of sulfated substrates; galacto configured monosaccharides and an unknown sulfate conjugate which is enriched in the marine environment. Alanine scanning was carried out on the 0 subsite of BT1624 ${ }^{6 \mathrm{~S}-\mathrm{Gal} / \mathrm{GalNAc}}$ to further inform its carbohydrate binding interactions but no kinetic data could be obtained. Qualitative analysis by TLC and HPAEC however, revealed that the mutants were active but that against 6S-Gal mutation of any residues in the 'recognition triad' resulted in either complete loss, or trace levels of activity, whilst against 6S-GalNAc only the D170A mutant resulted in complete loss of activity (Figure 5c and S6a). DSF analysis indicates that all proteins were folded but were less stable, with exception of R171A which showed an increase in stability (Figure S6b). As expected, the mutants bound Gal more weakly than the wildtype BT1624 ${ }^{6 S-G a l / G a l N A c}$ (Figure S6c).

A key difference between the structures of the 4 S1_15 enzymes is in the openness of their respective subsites (Figure 5b). BT46316s-Gal/GalNAc displays a more open S subsite with the C6 sulfate solvent exposed and the 0 subsite may allow the accommodation of additional groups. It is likely that true substrate targeted by this sulfatase remains to be discovered. In the other 3 structures, an lle is found over the $S$ subsite and the sulfate group is buried in a deep pocket, whilst further differences in the residues interacting with $\mathrm{C} 2$ substituents drive variation in substrate preference. BT1624 $4^{6 S-G a l / G a l N A c}$ forms few or no interactions with the $N$-acetyl group of GalNAc or the $\mathrm{O} 2$ of Gal and has a more open pocket than BT33336S-GalNAc (Figure 5a, b). Indeed, BT1624 ${ }^{6 S-G a l / G a l N A c}$ displays equal affinity, and activity, on both 6S-Gal and 6S- 
234 GalNAc $^{10}$ (Table S5). By contrast, in BT3333 ${ }^{6 S-G a l N A c}$ W464 is positioned to interact with the $N$-acetyl group of GalNAc through a stacking interaction with Y463 (Figure 5a). This leads to the enzyme having both a greater affinity and activity on 6S-GalNAc than BT1624 ${ }^{6 S-G a l / G a I N A c}$, and no detectable activity on 6S-Gal (Table S5) ${ }^{10}$. Mutation of W464 to Ala, or deletion of Y463/W464, significantly reduces the preference of BT33336s-GalNAc for GalNAc, whilst slightly increasing its interaction with Gal (Figure 5d). It is interesting to note that W464 is also present in BT4631 ${ }^{6 S-G a l / G a l N A c}$ (W469) but the Y463 is replaced by T464, a less bulky amino acid (Figure 5a). In the absence of the positioning aromatic, W469 flips down and is unable to interact with the $N$-acetyl of the 0 subsite sugar. Additional bioinformatic analysis of S1_15 enzymes located within Bacteroides PULs targeting chondroitin sulfate (CS), showed a retention of W464. However, Y463 is only partially retained, being mainly replaced by the aromatic residues Phe and His, which are also capable of stacking against W464 thus, preserving its functionality to interact with the $N$-acetyl group of GalNAc (Extended data 2). Together these data suggest that the sulfatases located within CS PULs have evolved to specifically target 6S-GalNAc. In comparison to the other 3 S1_15 enzymes, BT31096S-Gal forms an additional interaction with the $\mathrm{O} 2$ of Gal via the carboxy terminus of K508 (Figure 5a, b). This interaction appears to exclude the $N$ acetyl group from binding explaining the strong preference for 6S-Gal over 6S-GaINAc substrates, as indicated by activity and affinity studies (Figure 5e and Table S5). To further confirm this result, we generated a mutant $\left(B T 3109^{\Delta C T}\right)$ where we removed the carboxy terminus region (VEEEPLK) of BT31096S-Gal. The BT3109 ${ }^{\Delta C T}$ mutant did not show a preference for Gal and bound both Gal and GalNAc with a similar affinity (Figure 5e). This indicates that the terminal region of BT31096S-Gal tailors substrate specificity towards Gal. Interestingly, the VEEEPLK sequence is only found in 50 other sequences analysed and is found almost exclusively in S1_15 sulfatases from Bacteroidetes inhabiting marine environments (Table S6). utilise D361/D385 and R363/R387 to coordinate the O4 through $\mathrm{O} \delta 2$, and $\mathrm{N} \eta 1$, respectively, and $\mathrm{H} 445 / \mathrm{H} 471$ to coordinate $\mathrm{O} 3$ via $\mathrm{N} \varepsilon 2$ (Figure 6). The key role of 
subsite of BT31776S-GlcNAc (Table S5). The D361A mutation causes complete loss of activity whilst R363A and H445A cause $\sim 15$ and $\sim 1500$-fold loss in catalytic activity, respectively (Table S5). The Asp, Arg, and His are found within conserved motifs and are present in $91 \%$ of all 955 representative sequences analysed, with Asp and Arg even higher at 98 and 99\% (Extended data 3 and Table S7). This suggests that the S1_11 subfamily may exclusively target 6S-D-gluco configured carbohydrates.

BT3177 ${ }^{6 S-G I C N A c}$ and BT4656 6 S-GIcNAc/GIcNS have no discernible differences in the residues recognising GlcNAc/GlcNS unless focus is directed toward the amino acids coordinating the $N$-acetyl group and the $N$-sulfate groups, respectively. This region shows high amino acid diversity and is absent in around half of the S1_11 sequences analysed (Extended data 3 and Table S7). BT31776S-GlcNAc hydrogen bonds to the carbonyl of the $\mathrm{N}$-acetyl group through the phenol $\mathrm{OH}$ of $\mathrm{Y} 250$, and the overall environment has a hydrophobic character having L263 and L266 sitting above Y250 (Figure 6a). Mutation of Y250 to Ala caused a $~ 10$-fold loss in activity whilst mutation to Phe had no effect demonstrating that only the hydrophobic character of the aromatic phenyl ring is important (Table S5). By contrast, BT4656 6S-GIcNAc/GICNS has W273 and R290 situated in this region, whilst L263 is absent leaving a more open landscape (Figure 6b). R290 sits above W273 with the two residues interacting through cation$\pi$ interactions, positioning R290 to form a bidentate ionic interaction with the $N$-sulfate group, whilst the $\mathrm{N} \varepsilon 1$ of the Trp indole ring forms a hydrogen bond with the third oxygen of the sulfate. This gives this area of the protein a far more basic charge as compared to BT3177 ${ }^{6 S-G I C N A c}$ (Figure 6). Despite the high variability of this region (Extended data 3 and Table S7), homologues of BT4656 ${ }^{6 S-G I C N A c / G I C N S}$, harbouring W273/R290, are highly conserved in PULs targeting the glycans Hep/HS where 6SGlcNS is enriched compared to other sulfated substrates (Extended data 4). Although, BT4656 6 -GIcNAc/GICNS is active on 6S-GlcNAc, also found in Hep/HS, the presence of R290/W273 may provide enhanced activity towards $2 \mathrm{~N}$-sulfated $6 \mathrm{~S}$ GIcNAc compared to BT3177 $6 \mathrm{~S}-$ GIcNAc. Indeed, although the $k_{\text {cat }} / K_{\mathrm{M}}$ is similar for both substrates (Table S5), DSF analysis shows that BT4656 6 S-GIcNAc/GIcNS has a greater affinity for GlcNS than GIcNAc, whilst the reciprocal is true for BT3177 ${ }^{6 \mathrm{~S}-G I c N A c}$ (Figure 6b). This result is consistent with BT3177 6 S-GICNAc being encoded in a PUL associated with mucin degradation ${ }^{10}$ and therefore more tailored towards 6 S-GlcNAc, a sulfated linkage common in mucin O-glycans. 

that around $50 \%$ come from a marine origin whilst, S1_11 has a 2-3 fold enrichment in sequences ( $27 \%$ total) from a terrestrial/soil based environment when compared to the S1_15, S1_16, and S1_46 subfamilies (Table S7). Interestingly, despite 6SGlcNAc being a common component of host glycans this subfamily contains relatively few sequences (15\%) from the host environment. Conversely, 6S-gluco configured substrates are not well associated with marine environments. This observation suggests that marine S1_11 sulfatases utilise the invariant recognition triad to target a different substrate to the S1_11 sulfatase characterised here or to target an unknown substrate rich in 6S-gluco configured sugars.

\section{Discussion}

312 CAZymes are exquisitely specific enzymes, often distinguishing between single epimeric features to drive their specificity. S1 carbohydrate sulfatases are no exception to this. S1_11 and S1_15 subfamilies utilise an identical 'recognition triad' but specificity for GIcNAc and GalNAc (epimeric at 04 ) is achieved by the residues coming from the C-terminus in S1_11 and the N-terminus in S1_15, meaning no interactions are spatially conserved and thus recognition is specific (see supplemental discussion, Figure S7, and Table S8 for further comparison). The finer details of recognition, however, are 'hidden' in the non-conserved, and highly variable, area of the proteins. In these regions BT3177 ${ }^{6 S-G I C N A c}$ and BT3333 ${ }^{6 S-G a l N A c}$ have both evolved aromatic residues capable of interacting with the $\mathrm{N}$-acetyl group, of their respective substrates. BT3177 ${ }^{6 S-G I C N A c}$ resides in a PUL targeting mucin O-glycans where 6SGlcNAc is a common component, whilst BT3333 ${ }^{6 S-G a l N A c}$ resides in a PUL targeting CS where 6S-GaINAc can comprise up to $50 \%$ of the polymer (Figure 1). By contrast BT16246S-Gal/GalNAc, also in a PUL targeting mucin O-glycans, shows no tailored adaptation in this region, and as such has similar activity on both $6 \mathrm{~S}-\mathrm{Gal}$ and $6 \mathrm{~S}-$ GalNAc. Finally, BT4656 $65-G 1 c N A c / G I c N S$, which resides in a PUL targeting the GAGs Hep and $\mathrm{HS}$, has a positively charged nature in the equivalent area. This provides a stronger interaction with the doubly sulfated 6S-GIcNS, a component found exclusively in Hep and HS. Interestingly, GAGs have been shown to be high priority substrates for several Bacteroides species ${ }^{17}$ and the enhanced activities of sulfatases targeting host glycans may bestow a critical advantage on these substrates within the competitive gut environment. 
BT31096s-Gal shows an unusual C-terminal feature where the C-terminal carboxyl group caps the active site excluding the ability to recognize GalNAc. BT31096S-Gal resides in a PUL of unknown function but also contains a GH2 and GH43_31, both of which can target Gal in the pyranose and furanose form, respectively. Therefore, combined with the knowledge that BT31096S-Gal orthologues are most commonly found in marine environments, we suggest that this PUL may target a marine polysaccharide containing $6 \mathrm{~S}-\mathrm{Gal}$, an example of which being $\lambda$ carrageenan or potentially a more enigmatic marine glycan not yet identified ${ }^{25}$. Indeed, the ability of the human gut microbiota to metabolise marine glycans was recently revealed to be more extensive than previously thought ${ }^{26}$ suggesting these glycans may make a significant contribution to the colonic ecosystem.

The S1_46 subfamily sequences from both Firmicutes and Bacteroidetes inhabiting the human gastrointestinal tract require the $\mathrm{N}$-acetyl group for activity. All the key residues are invariant with the exception of Y408 that is preserved in Bacteroidetes but is mostly absent in gut Firmicutes. Intriguingly, these recognition features were also found in several pathogens suggesting that S1_46 sulfatases could be important enzymes for accessing host glycans in these species. For S1_46 sequences from the marine environment significant variability is seen in the key residues recognising the $\mathrm{N}$-acetyl group suggesting this not a feature encountered in their substrate. The sequences from the gastrointestinal tract most closely related to those from the marine environment also display similar mutations thus, despite their differing environments these sequences may target a similar, as yet unidentified, substrate to their marine counterparts. This observation further strengthens support for recent data highlighting marine glycan metabolism within the HGM.

The enrichment of sequences from a terrestrial/soil based environment in S1_11 is intriguing due to the presence of 6S-GIcNAc in nodulation (NOD) factors; bacterially produced lipooligosaccharides essential for bacteria to invade plant roots and establish nitrogen fixing nodules ${ }^{27}$. Indeed, several bacterial S1_11 sequences were isolated from rhizosphere communities and root nodules suggesting that S1_11 sulfatases could be involved in NOD factor metabolism. Interestingly, S1_11 sequences within the fungal phylum Ascomyota are well represented in plant pathogens. This alludes to a potential dichotomy where plant associated bacterial species may utilise S1_11 sulfatases to de-sulfate NOD factors in a positive manner, 
whilst S1_11 sequences in fungal Ascoymota may act as virulence factors, potentially by hijacking the NOD factor system. It should be noted however that the S1_11 sulfatases here are exo-acting, from the non-reducing end, and that NOD factors are sulfated at the reducing end of their chito-oligosaccharide component. This does not readily lend itself to a mechanism where $\mathbf{S 1} 11$ sulfatases act directly on intact NOD factors but these sulfatases may instead be involved in their downstream catabolism.

\section{Conclusion}

S1 carbohydrate sulfatases are exquisitely tailored enzymes deriving their binding energy and specificity from the nature of the glycan to which the target sulfate is appended. This makes these enzymes excellent targets for small molecule intervention to modify their function, develop tools to probe sulfated glycan metabolism, and disease intervention where sulfated glycan metabolism is perturbed.

\section{Materials and methods}

\section{Recombinant Protein Production.}

Genes were amplified by PCR using the appropriate primers and the amplified DNA cloned into $\mathrm{pET} 28 \mathrm{~b}$ using $\mathrm{Nhel} / \mathrm{Xhol}$ restriction sites generating constructs with $\mathrm{N}$ terminal His6 tags (Supplementary Table 11). Recombinant genes were expressed in Escherichia coli strains BL21 (DE3) or TUNER (Novagen), containing the appropriate recombinant plasmid, and cultured to mid-exponential phase in LB supplemented with $50 \mu \mathrm{g} / \mathrm{mL}$ kanamycin at $37^{\circ} \mathrm{C}$ and $180 \mathrm{rpm}$. Cells were then cooled to $16{ }^{\circ} \mathrm{C}$, and recombinant gene expression was induced by the addition of $0.1 \mathrm{mM}$ isopropyl $\beta-D-1-$ thiogalactopyranoside; cells were cultured for another $16 \mathrm{~h}$ at $16{ }^{\circ} \mathrm{C}$ and $180 \mathrm{rpm}$. The cells were then centrifuged at 5,000 $\times \mathrm{g}$ and resuspended in $20 \mathrm{mM}$ Hepes, $\mathrm{pH} 7.4$, with $500 \mathrm{mM} \mathrm{NaCl}$ before being sonicated on ice. Recombinant protein was then purified by immobilized metal ion affinity chromatography using a cobalt-based matrix (Talon, Clontech) and eluted with $100 \mathrm{mM}$ imidazole. For the proteins selected for structural studies, another step of size exclusion chromatography was performed using a Superdex 16/60 S75 or S200 column (GE Healthcare), with 10 mM HEPES, $\mathrm{pH} 7.5$, and $150 \mathrm{mM} \mathrm{NaCl}$ as the eluent, and they were judged to be $\geq 95 \%$ pure by SDS-PAGE. Protein concentrations were determined by measuring absorbance at 
400

401

402

403

404

405

406

407

408

409

410

411

412

413

414

415

416

417

418

419

420

421

422

423

424

425

426

427

428

429

430

431

432

$280 \mathrm{~nm}$ using the molar extinction coefficient calculated by ProtParam on the ExPasy server (web.expasy.org/protparam/).

Site-Directed Mutagenesis. Site-directed mutagenesis was conducted using the PCR-based QuikChange kit (Stratagene) according to the manufacturer's instructions using the appropriate plasmid as the template and appropriate primer pairs (Table S9).

\section{Microfuidics de-sulfation assays}

Sulfated carbohydrates were labelled at their reducing end with BODIPY which has a maximal emission absorbance of $\sim 503 \mathrm{~nm}$, which can be detected by the EZ Reader via LED-induced fluorescence. Non-radioactive microfluidic mobility shift carbohydrate sulfation assays were optimised in solution with a 12-sipper chip coated with CR8 reagent and a PerkinElmer EZ Reader II system using EDTA-based separation buffer and real-time kinetic evaluation of substrate de-sulfation. Pressure and voltage settings were adjusted manually (1.8 psi, upstream voltage: $2250 \mathrm{~V}$, downstream voltage: $500 \mathrm{~V}$ ) to afford optimal separation of the sulfated and unsulfated product with a sample (sip) time of $0.2 \mathrm{~s}$, and total assay times appropriate for the experiment. Individual de-sulfation assays were carried out at $28^{\circ} \mathrm{C}$ and assembled in a 384-well plate in a volume of $80 \mu \mathrm{l}$ in the presence of substrate concentrations between 0.5 and $20 \mu \mathrm{M}$ with $100 \mathrm{mM}$ Bis-Tris-Propane or Tris, depending on the $\mathrm{pH}$ required, $150 \mathrm{mM}$ $\mathrm{NaCl}, 0.02 \%(\mathrm{v} / \mathrm{v})$ Brij-35 and $5 \mathrm{mM} \mathrm{CaCl}$. The degree of de-sulfation was directly calculated using the EZ Reader software by measuring the sulfated carbohydrate: unsulfated carbohydrate ratio at each time-point. The activity of sulfatase enzymes was quantified in 'kinetic mode' by monitoring the amount of unsulfated glycan generated over the assay time, relative to control assay with no enzyme; with sulfate loss limited to $\sim 20 \%$ to prevent of substrate and to ensure assay linearity. $k_{c a t} / K_{M}$ values, using the equation $V_{0}=\left(V_{\max } / K_{M}\right)[S]$, were determined by linear regression analysis with GraphPad Prism software. Substrate concentrations were halved and doubled to check linearity of the rates ensuring substrate concentrations were significantly $<K_{\mathrm{M}}$. 


\section{HPAEC and TLC sulfatase enzymatic assays}

For reactions analysed by thin layer chromatography (TLC) $2 \mu \mathrm{L}$ of each sample was spotted onto silica plates and resolved in butanol:acetic acid:water (2:1:1) running buffer. The TLC plates were dried, and the sugars were visualized using diphenylamine stain $(1 \mathrm{ml}$ of $37.5 \% \mathrm{HCl}, 2 \mathrm{ml}$ of aniline, $10 \mathrm{ml}$ of $85 \% \mathrm{H} 3 \mathrm{PO}, 100 \mathrm{ml}$ of ethyl acetate and $2 \mathrm{~g}$ diphenylamine) and heated at $450^{\circ} \mathrm{C}$ for $2-5 \mathrm{~min}$ with a heat gun. Where possible, the enzymatic activity was confirmed by high-performance anionic exchange chromatography (HPAEC) with pulsed amperometric detection using standard methodology. The sugars (reaction products) were bound to a Dionex CarboPac PA200 column and eluted with an isocratic flow of $80 \mathrm{mM} \mathrm{NaOH}$ for $15 \mathrm{~min}$, the column was then cleaned with $500 \mathrm{mM} \mathrm{NaOH}$ for 10 min before being ran back into $80 \mathrm{mM} \mathrm{NaOH}$ at a flow rate of $0.25 \mathrm{ml} \mathrm{min}^{-1}$ before injection of the next sample. The reaction products were identified using the appropriated standards.

\section{Differential scanning fluorimetry}

Thermal shift/stability assays (TSAs) were performed using a StepOnePlus Real-Time PCR machine (LifeTechnologies) and SYPRO-Orange dye (emission maximum 570 $\mathrm{nm}$, Invitrogen) as previously described ${ }^{28}$ with thermal ramping between 20 and $95^{\circ} \mathrm{C}$ in $0.3^{\circ} \mathrm{C}$ step intervals per data point to induce denaturation in the presence or absence of various carbohydrates as appropriate to the sulfatase being analysed. The melting temperature ( $\mathrm{Tm}$ ) corresponding to the midpoint for the protein unfolding transition was calculated by fitting the sigmoidal melt curve to the Boltzmann equation using GraphPad Prism, with $\mathrm{R}^{2}$ values of $>0.99$. Data points after the fluorescence intensity maximum were excluded from the fitting. Changes in the unfolding transition temperature compared with the control curve $\left(\Delta T_{m}\right)$ were calculated for each ligand. $A$ positive $\Delta T_{m}$ value indicates that the ligand stabilises the protein from thermal denaturation, and confirms binding to the protein. All TSA experiments were conducted using a final protein concentration of $5 \mu \mathrm{M}$ in $100 \mathrm{mM}$ Bis-Tris-Propane independent assays were performed for each protein and protein ligand combination 


\section{Glycan labelling}

469 Sulfated saccharide samples were labelled according to a modification of the method 470 by Das et al., reporting the formation of $\mathrm{N}$-glycosyl amines for 4,6-O-benzilidene 471 protected D-gluopyranose monosaccharides with aromatic amines ${ }^{29}$. Briefly, the 472 lyophilised sugar (1 mg) was dissolved in anhydrous methanol $(0.50 \mathrm{~mL}$, Sigma473 Aldrich) in a $1.5 \mathrm{~mL}$ screw-top PTFE microcentrifuge tube and BODIPY-FL hydrazide 474 (4,4-difluoro-5,7-dimethyl-4-bora-3a,4a-diaza-s-indacene-3-propionic acid, 475 hydrazide, $0.1 \mathrm{mg}$, ThermoFischer, $\lambda$ ex./em. 493/503, $\varepsilon 80,000 \mathrm{M}^{-1} \mathrm{~cm}^{-1}$ ) was added and the mixture vortexed $(1 \mathrm{~min})$, then reacted $\left(65^{\circ} \mathrm{C}, 24 \mathrm{~h}\right)$ in darkness. The products were then cooled and a portion purified by TLC on silica coated aluminium plates (silica gel 60, Sigma-Aldrich, Millipore) developed with methanol or 1:1 v/v ethyl acetate/methanol. The unreacted BODIPY-FL label (orange on the TLC plate) was identified by reference to a lane containing the starting material (BODIPY-FL hydrazide), allowing differentiation from the putative labelled product (also orange). This latter band (which can run ahead or behind the label depending on the sugar; e.g. labelled GlcNAc $4 S$ and $6 S$ both run with $R_{f} 0.84$ compared to label, $R_{f} 0.70$; others, such as labelled GalNAc4S or $6 \mathrm{~S}$ require, 1:1 v/v ethyl acetate/methanol and the product runs behind the label on TLC) was scraped from the plates and extracted in fresh methanol ( $2 \times 0.5 \mathrm{~mL})$, spun (benchtop centrifuge, 3 minutes), the supernatant recovered and dried (rotary evaporator) to afford the fluorescent, coloured product (bright green in aqueous solution), which was then employed in subsequent experiments.

Isothermal Calorimetry (ITC).

493 The affinity of BT33336S-GalNAc and BT4656 ${ }^{6 S-G I C N A c / G I C N S}$ against $6 S-G a I N A c$ and $6 S-$ 494 GlcNAc, respectively, was quantified by ITC using a Microcal ITC ${ }^{200}$ calorimeter. The 495 protein samples (70 $\mu \mathrm{M}$ for BT3333 ${ }^{6 \mathrm{~S}-G a l N A c}$ and $60 \mu \mathrm{M}$ for BT4656 ${ }^{6 \mathrm{~S}-G I c N A c / G I c N S}$ ), stirred at $400 \mathrm{rpm}$ in a $0.2-\mathrm{mL}$ reaction cell, was injected with $182 \mu \mathrm{L}$ aliquots of ligand, preceded by 1 injection of $0.2 \mu \mathrm{L}$ with a delay of 180 seconds between injections $(0.8$ mM 6S-GaINAc was used for BT33336S-GalNAc and $0.4 \mathrm{mM} \mathrm{6S-GIcNAc}$ for BT4656 6 S- 
500

501

502

503

504

505

506

507

508

509

510

511

512

513

514

515

516

517

518

519

520

521

522

523

524

525

526

527

528

529

530

531

532

533

Integrated binding heats minus dilution heat controls were fit to a single set of sites binding model to derive $K_{\mathrm{A}}, \Delta \mathrm{H}$, and $\mathrm{n}$ (number of binding sites on each molecule of protein) using Microcal Origin v7.0.

\section{Static light scattering determination of molecular weight}

Molecular weights were determined using an Agilent Multi-Detector System calibrated with bovine serum albumin. Proteins were separated by size exclusion chromatography using an Agilent BioSEC Advance $300 \AA$, 4.6 × $300 \mathrm{~mm}$ or GE Superdex $\begin{array}{lllllll}200 & 10 & 300 & \text { columns equilibrated with } 20 & \mathrm{mM}\end{array}$ tris(hydroxymethyl)aminomethane- $\mathrm{HCl}$ pH 7.4, $150 \mathrm{mM} \mathrm{NaCl}$ buffer. Light scattering data was collected at $90^{\circ}$ and refractive index used to calculate absolute molecular weight.

\section{Crystallisation of carbohydrate sulfatases.}

After purification, all proteins were concentrated in centrifugal concentrator with a molecular weight cutoff of $30 \mathrm{KDa}$ in the size exclusion chromatography buffer. Sparse matrix screens were set up in 96-well sitting drop SPT Labtech plates plates (400-nL drops). Initial hits crystals for all proteins were obtained between 20 and $35 \mathrm{mg} / \mathrm{mL}$ supplemented with between 10 and $30 \mathrm{mM}$ ligand unless otherwise stated. For all sulfatase the wildtype $B$. theta variants were used, having a Ser at the catalytic formylglycine position. BT16246S-Gal/GalNAc with 6S-GalNAc crystallised in $20 \%$ Polyethyleneglycol (PEG) 6000, 0.2 M ammonium chloride and $0.1 \mathrm{M}$ sodium acetate pH 5.5. BT1918 ${ }^{3 S-G I c N A c}$ with 6S-GIcNAc crystallised in $45 \%$ Methylpentanediol (MPD) $0.2 \mathrm{M} \mathrm{CaCl}_{2}$ and $0.1 \mathrm{M}$ Bis-Tris pH 5.5. BT30574S-Gal/GalNAc with 4S-GalNAc crystallised in $20 \%$ PEG 3350 and 0.2 M sodium nitrate. BT31096S-Gal with 6S-Gal crystallised in $30 \%$ PEG 4000, $0.2 \mathrm{M}$ ammonium acetate and sodium citrate $\mathrm{pH}$ 5.6. BT31776S-GIcNAc with 6S-GlcNAc crystallised in $50 \%$ precipitant mix 1 (40\% v/v PEG 500 MME; $20 \%$ w/v PEG 20000), 0.1 M carboxcylic acids (0.2 M Sodium formate; 0.2 M Ammonium acetate; $0.2 \mathrm{M}$ Sodium citrate tribasic dihydrate; $0.2 \mathrm{M}$ Sodium potassium tartrate tetrahydrate; $0.2 \mathrm{M}$ Sodium oxamate) and $0.1 \mathrm{M}$ buffer system $3 \mathrm{pH} 8.5$ (Tris (base); BICINE). BT37964S-Gal/GaINAc with 4S-GaINAc crystallised in $20 \%$ PEG 6000, 0.2 M magnesium chloride and $0.1 \mathrm{MES} \mathrm{pH} 6.0$. BT46316S-Gal/GalNAc was crystallised at 80 $\mathrm{mg} / \mathrm{ml}$ with $10 \mathrm{mM}$ 6S-Gal in $20 \%$ PEG 10000 with $0.1 \mathrm{M}$ Bicine pH 8.5. All crystals were cryo-cooled with the addition of the ligand they were crystallised with. $20 \%$ PEG 
400 was used as the cryoprotectant for BT1624 ${ }^{6 S-G a l / G a l N A c}$, BT3057 ${ }^{4 S-G a l / G a l N A c}$, and BT31096s-Gal and $100 \%$ paratone-N oil for BT37964S-Gal/GalNAc. PEG 200 was used as cryoprotectant for BT46316S-Gal/GalNAc crystals. No cryoprotectant was added to BT1918 3 S-GICNAC or BT31776S-GICNAc crystals as the crystallisation condition afforded sufficient cryoprotection. Data were collected at Diamond Light Source (Oxford) on beamlines 103, 104, 104-1 and I24, and SOLEIL on the PROXIMA_1 beamline at 100 $\mathrm{K}$. The data were integrated with $\mathrm{XDS}^{30}$, or Xia2 3di or 3dii, and scaled with Aimless $^{31,32}$. Five percent of observations were randomly selected for the $R_{\text {free }}$ set. The phase problem was solved by molecular replacement using the automated molecular replacement server Balbes ${ }^{33}$ for all proteins except BT31096S-Gal and BT46316SGal/GalNAc which were solved using Phase ${ }^{34}$ and BT1624 ${ }^{6 S-G a l / G a l N A c}$ as the search model after preparation with sculptor. Models underwent recursive cycles of model building in $\operatorname{Coot}^{35}$ and refinement cycles in Refmac5 ${ }^{36}$. Where necessary ligand restraint and coordinates were generated with Jligand ${ }^{37}$. The models were validated using Coot and MolProbity ${ }^{38}$. Structural Figures were made using Pymol (The PyMOL Molecular graphics system, Version 2.0 Schrodinger, LLC.) and all other programs used were from the CCP4 suite ${ }^{39,40}$. The data processing and refinement statistics are reported in Table S12 and S13.

552

\section{Global phylogenetic trees of S1_11, S1_15, S1_16, S1_46 sequences.}

On the basis of the taxonomic diversity, to avoid identical sequences we selected a representative number of sequences within each subfamily: a) for S1_11, 955 sequences were selected among the 2177 sequences present in the subfamily S1_11 from the SulfAtlas database and 411 positions were used for phylogeny; b) for S1_15, 920 sequences were selected among the 1906 sequences present in SulfAtlas and 365 positions were used for phylogeny; c) for S1_16, 800 sequences were selected among the 1361 sequences present in Sulfatlas and 342 positions were used for phylogeny; and d) for S1_46, 349 out of the total 574 sequences present in Sulfatlas were selected, 401 positions were used for phylogeny. In each case, the sequences were aligned by MAFFT v.7 $7^{41}$ using L-INS-i algorithm. The multiple sequence alignments were visualized by Jalview software v.11.042, non-aligned regions were removed, and the above listed respective numbers of positions were used for the phylogeny. Phylogeny was made using RAxML v. 8.2.4 ${ }^{43}$. The phylogenetic tree was 
using a discrete Gamma distribution to model evolutionary rate differences among sites (4 categories). The rate variation model allowed for some sites to be evolutionarily invariable. The reliability of the trees was tested by bootstrap analysis using 1000 resamplings of the datase ${ }^{46}$. In all cases, fifteen S1_0 sequences from the sulfAtlas database were used as outgroup.

\section{Data availability statement}

Source Data for all experiments, along with corresponding statistical test values, where appropriate, are provided within the paper and in Supplementary information. The crystal structure dataset generates have been deposit in the in the Protein Data Bank (PDB) under the following accession numbers: 7OZ8, 7OZ9, 7OZA, 7OZE, 7OZC, 7P26, and 7P24.

\section{Code availability statement}

No new codes were developed or compiled in this study

\section{Competing interests statement}

The authors declare no competing interests.

\section{Acknowledgements}

This project has received funding from the European Union's Horizon 2020 research and innovation programme under the Marie Skłodowska-Curie grant agreement № 748336, the European Research Council ERC (694181), The Knut and Alice Wallenberg Foundation (2017.0028), Swedish Research Council (2017-00958), Wilhelm och Martina Lundgrens Vetenskapsfond (2020.3597, awarded to ASL) and the Academy of Medical Sciences/Wellcome Trust through the Springboard Grant SBF005\1065 163470 awarded to AC. The authors acknowledge access to the SOLEIL and Diamond Light sources via both the University of Liverpool and Newcastle University BAGs (proposals m×21970 and mx18598, respectively). We thank the staff of DIAMOND, SOLEIL, and members of the Liverpool's Molecular biophysics group for assistance with data collection. We are also grateful for Dr. Erwan Corre's help regarding bioinformatics analyses (ABIMS platform, Station Biologique de Roscoff, France). 


\section{Author contributions}

604

ASL, ECM, and AC designed experiments and wrote the manuscript.

605

ASL and AC cloned, expressed, purified sulfatases and performed the enzymatic

606 assays.

607 AC, DPB, JAL, and PAE carried out and analysed kinetic and binding experiments

608 EY and JAL performed labelling and NMR experiments

$609 A C$ and $A B$ performed structural biology experiments.

$610 \mathrm{MC}$ and TB performed sulfatase phylogenetic analyses.

611 GW carried out light scattering experiments and size determination.

612 All authors read and approved the manuscript.

\section{Figure 1. Examples of sulfated host carbohydrates found in the colon}

615 Schematic representation of sulfated host glycans found in mucin O-glycans (green 616 box) and glycosaminoglycans which are integral to the extracellular matrix and 617 glycocalyx (peach box), representing a constant, host derived, nutrient source for the 618 colonic microbiota. Sugars are shown according to the Symbol Nomenclature for Glycan system ${ }^{47}$.

620

\section{Figure 2. Conserved features of S1 carbohydrate sulfatases.}

622 a, Example of the overall fold common to all S1 sulfatases, Cartoon representation of BT3177 6 S-GICNAc colour ramped from blue $(\alpha / \beta / \alpha \mathrm{N}$-terminal domain) to red ( $\beta$-sheet $\mathrm{C}$ terminal domain). The black dashed circle indicates the C-terminal sub-domain and the red dashed circle the active site location on the core $\alpha / \beta / \alpha$ domain. $b$, Conservation of the consensus active site sequence for S1_11, S1_15, S1_16 and S1_46. The position of formylglycine installation is highlighted with a grey box $\mathbf{c}$, Examples of the calcium binding site: left side (green), representation of BT31776S-GICNAc calcium site as an example of the typical site observed in most sulfatase structures described to date; right side, display of alternate sites observed in BT1918 3 S-GIcNAc (yellow) and BT1596 $2 \mathrm{~S}-\triangle 4,5 \mathrm{UA}$ (cyan). The variable areas are highlighted in blue and red dashed circles. 

interactions of the 0 subsite of BT1918 ${ }^{3 \mathrm{~S}-\text { GICNAc }}$ (left panel) and surface representation of the structural data for BT1918 ${ }^{3 S-G l c N A c}$ showing the 0 subsite pocket (right panel). Highlighted in green are the residues interacting with the $\mathrm{N}$-acetyl, in blue are the sulfate flanking residues, and orange indicates sugar ring only interactions. b,

642 Catalytic effects of alanine scanning on BT1918 ${ }^{3 S-G I c N A c}$. Percentages above the bar indicate relative activity to wildtype (WT). c, Radial version of the phylogenetic tree of representative sulfatases from subfamily S1_46. For clarity all labels and sequence accession codes have been omitted. The annotations next to the colour code concern the presence or absence of conservation of the residues crucial in substrate recognition by BT1918 ${ }^{3 S-G I c N A c}$ (acc-code Q8A6G6) and in the following order: Y94, N174, R327 and Y408. The residues are coloured as following: black means an equivalent residue is present; a grey and bold letter at any position means that the corresponding residue is replaced by that amino acid; a grey, bold and italic letter at any position means that the equivalent position is replaced by any type of amino acid; a bold grey letter followed by one-letter codes in parentheses indicates that the equivalent position can be substituted by any of those amino acids; the dash at the Y408-equivalent position indicates that no equivalent amino acid can be deduced from the multiple alignment. Branches having the same colour have the corresponding pattern in common. The red filled circle designates the sequence of the S1_46 sulfatase from $B$. thetaiotaomicron (See Figure S8 for full tree).

Figure 4. Structural details of the carbohydrate binding region of BT3057 4 S-Gal/GaINAc and BT37964S-Gal/GaINAc

Stick (left) and surface (middle) representation showing the carbohydrate binding interactions of the 0 subsite of S1_16 sulfatases: a, BT30574S-Gal/GalNAc and b, BT3796 4 S-Gal/GalNAc. The respective right panel show the effects of alanine scanning on these sulfatase activities with the percentages above the bar indicating the relative activity to wildtype (WT). c, Radial phylogenetic tree of representative sulfatases from subfamily S1_16. For clarity all labels and sequence accession codes have been omitted. The annotations next to the colour code concern the presence or absence of conservation of the critical residues in substrate recognition by BT30574S-Gal/GalNAc (acc-code Q8A397) in the order: W109, H182 and H423. Sequences coded by orange 
branches contain an additional W332 present in BT37964S-Gal/GalNAc (acc-code

671 Q8A171) but absent in other sequences. For simplification the residue numbers have 672 been omitted, except for W332. The residues are coloured as following: black means 673 an equivalent amino acid is present; a grey and bold letter at any position means that 674 the corresponding residue is replaced by that amino acid; a grey and italic letter at any 675 position means that the equivalent position is replaced by any type of amino acid; a 676 bold grey letter followed by one-letter codes in parentheses indicates that the 677 equivalent position can be substituted by any of those amino acids; the dash at the $\mathrm{H}$ 678 equivalent position indicates that no equivalent amino acid can be deduced from the 679 multiple alignment. When two patterns are indicated separated by a comma (i.e. W $680 \mathrm{H}, \mathrm{W} \mathrm{H}$-) both have been attributed the same colour code. Branches having the same 681 colour have the corresponding pattern in common. Red filled circles designate 682 sequences of S1_16 sulfatases from B. thetaiotaomicron (See Figure S9 for full tree).

Figure 5. Structural variations of the carbohydrate binding region of the S1_15 family

686

a, Stick and $\mathbf{b}$, Surface representation showing the carbohydrate binding interactions of the 0 subsite of BT46316S-Gal/GalNAc, BT16246S-Gal/GalNAc, BT33336S-GalNAc, and BT31096S-Gal (from top to bottom). c, High pressure anion exchange chromatography (HPAEC) of wildtype BT1624 ${ }^{6 S-G a l / G a l N A c}$ wildtype (WT) and its mutants. The produced product is highlighted by a grey box. HPAEC reactions utilised $6 \mathrm{mM}$ substrate and 5 $\mu \mathrm{M}$ enzyme, with $3 \mathrm{mM}$ HEPES, $45 \mathrm{mM} \mathrm{NaCl}$, and $5 \mathrm{mM} \mathrm{CaCl} 2$ over a $48 \mathrm{~h}$ period at $37^{\circ} \mathrm{C}$. d, DSF analysis of the effect of mutating the GalNAc specificity features of BT3333 ${ }^{6 S-G a I N A c}$. e, DSF analysis of the effect of mutating the C-terminal extension (VEEEPLK) which drives specificity towards Gal in BT31096S-Gal. 100 mM BTP pH 7.0 with $150 \mathrm{mM} \mathrm{NaCl}$ was used in all DSF experiments. Error bars represent s.e.m..

Figure 6. Structural variations of the carbohydrate binding region of the S1_11 family

a, Carbohydrate interactions of BT31776S-GICNAc. b, Carbohydrate interactions of BT4656 $6 \mathrm{~S}-\mathrm{GlcNAc/GlcNS}$. In both panels the left is a stick representation of the carbohydrate interactions at $\mathrm{SO}$, middle is a surface representation showing the local surface charge. c, DSF data showing the second derivative of the thermal melts with 
703

704

705

706

707

708

709

710

711

712

713

714

715

716

717

718

719

720

721

722

723

724

725

726

727

728

729

730

731

732

733

734

735

protein alone, $100 \mathrm{mM}$ GlcNAc, and $100 \mathrm{mM}$ GlcNS against $5 \mu \mathrm{M}$ BT31776S-GlcNAc (top), and $5 \mu \mathrm{M}$ of BT4656 6 S-GICNAc/GIcNS (bottom).

\section{Extended data 1. Radial version of the phylogenetic tree of representative} sulfatases from subfamily S1_15.

For clarity all labels and sequence accession codes have been omitted. The annotations next to the colour code concern the presence or absence of conservation of the indicated residues and in this order : I100, D170, R171, H220, K461 and A462. These residues are crucial in substrate recognition by BT1624 ${ }^{6 S-G a l / G a l N A c}$ (acc-code Q8A7A1). For simplification the residue numbers have been omitted. For example, an I in black means an equivalent isoleucine is present; a grey and bold letter at any position means that the corresponding residue is replaced by that amino acid; a grey and italic letter at any position means that the equivalent position can be replaced by any type of amino acid; a bold grey letter followed by one-letter codes in parentheses indicates that the equivalent position is substituted by any of those amino acids. Branches having the same colour have the corresponding pattern in common. Red filled circles designate sequences of S1_15 sulfatases from $B$. thetaiotaomicron (See Figure S10 for full tree).

\section{Extended data 2. Analysis of S1_15 enzymes with PULs targeting chondroitin} sulfate.

PULs targeting chondroitin sulfate aligned by orthologues of BT33336s-GalNAc. Light green background shows orthologues with Y463/W464, a dark green background highlights orthologues with F463/W464, a light blue back ground highlight orthologues with H463/W464, and a purple background highlight orthologues with Q463/W464. The numbering used corresponds to the sequence of BT33336s-GalNAc. A red background highlights the presence of GH88 and S1_27 (an endo 4S-chrondroitin sulfatase) which appear to be discrete genetic block not always physically localised to the PUL. A black background highlights a core block observed in CS PULs containing BT33336S-GalNAc orthologues. 

sulfatases from subfamily S1_11

738 For clarity all labels and sequence accession codes have been omitted. The annotations next to the colour code concern the presence or absence of conservation of the indicated residues and in this order: R290, W273, D385, R387 and H471. These residues are crucial in substrate recognition by BT4656 6 -GIcNAc/GIcNS (acc-code Q89YS5). For simplification the residue numbers have been omitted. For example, a $\mathrm{R}$ in black means an equivalent arginine is present; a grey and bold letter at this position means that the corresponding residue is replaced by that amino acid; the grey and italic $\mathrm{R}$ at this position means that the R-equivalent position is replaced by any type of amino acid; a bold grey $\mathrm{R}$ followed by one-letter codes in parentheses indicates that the R-equivalent position can be substituted by any of those amino acids; the dash at the R-equivalent position indicates that no equivalent amino acid can be deduced from the multiple alignment. Branches having the same colour have the corresponding pattern in common. Red filled diamonds designate sequences of S1_11 sulfatases from $B$. thetaiotaomicron. All sequences in the specific branch that contains BT4656 65 GICNAC/GICNS are found within a conserved heparin PUL (See Figure S11 for full tree).

Extended data 4. Analysis of S1_11 enzymes with PULs targeting chondroitin sulfate.

756

PULs targeting heparan sulfate (HS) aligned by orthologues of BT4656 ${ }^{6 S-G I c N A c / G I c N S}$. Orthologues of BT4656 ${ }^{6 S-G I c N A c / G l c N S}$ with W273/R290 and W273/Q290 are highlighted with green and blue background, respectively. A black background highlights a core block observed in HS PULs containing BT4656 6 -GIcNAc/GIcNS orthologues.

\section{Supplemental Figure 1. Extracted ligands and their electron density maps.}

762 The $2 m F_{\text {obs }}-F_{c}$ maps are shown contoured at $1 \sigma$ for all substrates and products cocrystallised with their respective sulfatase.

764

a, Right panel shows chromatogram of size exclusion chromatography coupled to light scattering for BT1918 3 S-GICNAC demonstrating the presence of a dimer(e= expected molecular mass; o=observed molecular mass). Left panel shows surface 
representation of the dimer of BT1918 3 S-GICNAc; the active site is highlighted with a black dashed circle. b, Thin layer chromatography analysis of BT1918 ${ }^{3 S-G l C N A C}$ and its mutants versus its $3 \mathrm{~S}, 6 \mathrm{~S}-\mathrm{N}$-acetylglucosamine substrate. Assays were ran for $48 \mathrm{~h}$, at $37^{\circ} \mathrm{C}$, deploying $6 \mathrm{mM}$ substrate and $5 \mu \mathrm{M}$ enzyme. All assays contained $3 \mathrm{mM}$ HEPES pH 7.0, $45 \mathrm{mM} \mathrm{NaCl}$, and $5 \mathrm{mM} \mathrm{CaCl}_{2}$. c, DSF analysis showing the stability of the mutant proteins of BT1918 ${ }^{3 S-G l C N A c}$ with respect to the wildtype (WT). d) Thin layer chromatography analysis of BT1918 ${ }^{3 \mathrm{~S}-\text { GICNAc }}$ versus 3S-glucosamine and 3S,6Sglucosamine. Assays were ran for $48 \mathrm{~h}$, at $37^{\circ} \mathrm{C}$, deploying $6 \mathrm{mM}$ substrate and $5 \mu \mathrm{M}$ enzyme. All assays contained $3 \mathrm{mM}$ HEPES pH 7.0, $45 \mathrm{mM} \mathrm{NaCl}$, and $5 \mathrm{mM} \mathrm{CaCl}$.

\section{Supplemental Figure 3. Gel chromatography and light scattering of S1_16} sulfatases.

Top panels are size exclusion chromatograms whilst, the middle and bottom panels are size exclusion coupled to light scattering chromatograms for the monomer (green) and dimer (black) peaks purified from size exclusion. $e=$ expected molecular mass; $\mathrm{o}=$ observed molecular mass.

\section{Supplemental Figure 4. Activity and stability analysis of S1_16 sulfatases and} their mutant variants.

a, DSF analysis of the effects of galactose and $N$-acetyl galactosamine on thermostability with a positive shift indicative of binding. $\mathbf{b}$, Normalised DSF melt curves of BT3057 4 S-Gal/GalNAc and BT37964S-Gal/GalNAc. c, DSF melt curves of the purified monomer and dimer species (left), monomer species in the presence of galactose and $\mathrm{N}$-acetyl galactosamine (middle), and the dimer species in the presence of galactose and $\mathrm{N}$-acetyl galactosamine (right). $\mathbf{d}$, Thin layer chromatography (TLC) analysis of wildtype (WT) and mutant S1_16 sulfatases. Asterisks are placed above lanes where activity is observed. e, High pressure anion exchange chromatography (HPAEC) of wildtype (WT) and mutant S1_16 sulfatases. A grey block highlights the produced product. Both TLC and HPAEC reactions utilised $6 \mathrm{mM}$ substrate and $1 \mu \mathrm{M}$ enzyme, except for W109A variants where $10 \mu \mathrm{M}$ was deployed, with $3 \mathrm{mM}$ HEPES, $45 \mathrm{mM}$ $\mathrm{NaCl}$, and $5 \mathrm{mM} \mathrm{CaCl} 2$ over a $48 \mathrm{~h}$ period at $37^{\circ} \mathrm{C}$. 
a, Chromatograms of size exclusion chromatography coupled to light scattering for coupled to light scattering for S1_11 subfamily members. $\mathrm{e}=$ expected molecular mass; o=observed molecular mass.

807

808

Supplemental Figure 6. Analysis of the activity and stability of BT1624 ${ }^{6 S-G a l / G a I N A c}$ and its mutant variants.

a, Thin layer chromatography (TLC) analysis of wildtype (WT) BT1624 ${ }^{6 S-G a l / G a l N A c}$ and its mutants. Asterisks are placed above lanes where activity is observed. Both TLC reactions utilised $6 \mathrm{mM}$ substrate and $5 \mu \mathrm{M}$ enzyme, with $3 \mathrm{mM} \mathrm{HEPES,} 45 \mathrm{mM} \mathrm{NaCl}$, and $5 \mathrm{mM} \mathrm{CaCl} 2$ over a $48 \mathrm{~h}$ period at $37^{\circ} \mathrm{C}$. b, DSF analysis showing the stability of the mutant proteins of BT1624 ${ }^{6 S-G a l / G a l N A c}$ with respect to the wildtype. c, DSF analysis of the effects of alanine scanning on the ability of BT1624 6 -Gal/GalNAc to bind galactose with the $T_{m}$ of the protein shown atop the bar. The experiments were performed with $5 \mu \mathrm{M}$ of protein and $324 \mathrm{mM}$ of galactose in $100 \mathrm{mM}$ BTP and $150 \mathrm{mM} \mathrm{NaCl}$.

Supplemental Figure 7. Biochemical comparison of S1_15 BT3333 ${ }^{6 S-G a I N A c}$ and

a, Isothermal titration calorimetry traces of inactive serine variants of BT3333 ${ }^{6 S-G a I N A c}$ and BT4656 6 -GIcNAc/GIcNS binding to their respective $6 \mathrm{~S}-$ GalNAc and $6 \mathrm{~S}-\mathrm{GICNAC}$ substrates. b, Specific activity of BT3333 ${ }^{6 S-G a I N A c}$ and BT4656 6 S-GIcNAc against $0.5 \mathrm{mM}$ their respective 6S-GalNAc and 6S-GIcNAc substrates, monitored by 1D H-NMR. c, Acid hydrolysis of $10 \mathrm{mM}$ 6S-GalNAc and 6S-GlcNAc in the presence of $250 \mathrm{mM} \mathrm{HCl}$ at $90^{\circ} \mathrm{C}$. subfamily S1_46.

830 The S1_46 subfamily is composed of 574 sequences (3 eukaryota, 5 Archaea and 566 Bacteria). To avoid identical sequences, and based on taxonomic diversity, 356 sequences were selected for alignment. 15 sequences that belong to the S1_0 subfamily (Phosphonate monoester hydrolase / phosphodiesterase) have been used 

subfamily S1_16.

837 The S1_16 subfamily is composed of 1356 sequences. To avoid the identical sequences, and based on taxonomic diversity, 800 representative sequences were used for the alignment. 15 sequences that belong to the S1_0 subfamily (Phosphonate monoester hydrolase / phosphodiesterase) have been used as outgroup for the alignment.

842

Supplemental Figure 10. Full Phylogenetic tree of representative sulfatases from subfamily S1_15.

The S1_15 sub-family is composed of 1895 sequences. To avoid the identical sequences, and based on taxonomic diversity 920 representative sulfatase sequence were selected. 15 sequences that belong to the S1_0 subfamily (Phosphonate monoester hydrolase / phosphodiesterase) have been used as outgroup for the alignment.

850

Supplemental Figure 11. Full Phylogenetic tree of representative sulfatases from subfamily S1_11.

853 Based on taxonomic diversity, 955 representative sulfatase sequences of 854 the S1_11 subfamily (44\% of the subfamily) was aligned. There are 15 sequences of the S1_0 subfamily, phosphonate monoesters, used as outgroup for phylogeny.

\section{References:}

1 Sender, R., Fuchs, S. \& Milo, R. Revised Estimates for the Number of Human and Bacteria Cells in the Body. Cheng, H. Y., Ning, M. X., Chen, D. K. \& Ma, W. T. Interactions Between the Gut Microbiota and the Host Innate Immune Response Against Pathogens. Front Immunol

8673 Belkaid, Y. \& Hand, T. W. Role of the microbiota in immunity and inflammation. Cell 157, 121-141, doi:10.1016/j.cell.2014.03.011 (2014).

8694 McNeil, N. I. The contribution of the large intestine to energy supplies in man. The American journal of clinical nutrition 39, 338-342, doi:10.1093/ajcn/39.2.338 (1984).

8715 Larsbrink, J. et al. A discrete genetic locus confers xyloglucan metabolism in select human gut Bacteroidetes. Nature 506, 498-502, doi:10.1038/nature12907 (2014). 
6 Luis, A. S. et al. Dietary pectic glycans are degraded by coordinated enzyme pathways in human colonic Bacteroides. Nature microbiology 3, 210-219, doi:10.1038/s41564017-0079-1 (2018).

7 Cartmell, A. et al. A surface endogalactanase in Bacteroides thetaiotaomicron confers keystone status for arabinogalactan degradation. Nature microbiology 3, 1314-1326, doi:10.1038/s41564-018-0258-8 (2018).

8 Cartmell, A. et al. How members of the human gut microbiota overcome the sulfation problem posed by glycosaminoglycans. Proceedings of the National Academy of Sciences of the United States of America 114, 7037-7042, doi:10.1073/pnas.1704367114 (2017).

9 Ndeh, D. et al. Metabolism of multiple glycosaminoglycans by Bacteroides thetaiotaomicron is orchestrated by a versatile core genetic locus. Nature communications 11, 646, doi:10.1038/s41467-020-14509-4 (2020).

10 Luis, A. S. et al. A single bacterial sulfatase is required for metabolism of colonic mucin $<$ em $>0</$ em $>$-glycans and intestinal colonization by a symbiotic human gut bacterium. bioRxiv, 2020.2011.2020.392076, doi:10.1101/2020.11.20.392076 (2020).

11 El Kaoutari, A., Armougom, F., Gordon, J. I., Raoult, D. \& Henrissat, B. The abundance and variety of carbohydrate-active enzymes in the human gut microbiota. Nat Rev Microbiol 11, 497-504, doi:10.1038/nrmicro3050 (2013).

12 Martens, E. C., Chiang, H. C. \& Gordon, J. I. Mucosal glycan foraging enhances fitness and transmission of a saccharolytic human gut bacterial symbiont. Cell host \& microbe 4, 447-457, doi:10.1016/j.chom.2008.09.007 (2008).

13 Terrapon, N., Lombard, V., Gilbert, H. J. \& Henrissat, B. Automatic prediction of polysaccharide utilization loci in Bacteroidetes species. Bioinformatics 31, 647-655, doi:10.1093/bioinformatics/btu716 (2015).

14 Goodman, A. L. et al. Identifying genetic determinants needed to establish a human gut symbiont in its habitat. Cell host \& microbe 6, 279-289, doi:10.1016/j.chom.2009.08.003 (2009).

$15 \mathrm{Li}, \mathrm{H}$. et al. The outer mucus layer hosts a distinct intestinal microbial niche. Nature communications 6, 8292, doi:10.1038/ncomms9292 (2015).

16 Tuncil, Y. E. et al. Reciprocal Prioritization to Dietary Glycans by Gut Bacteria in a Competitive Environment Promotes Stable Coexistence. mBio 8, doi:10.1128/mBio.01068-17 (2017).

17 Raghavan, V. \& Groisman, E. A. Species-specific dynamic responses of gut bacteria to a mammalian glycan. J Bacteriol 197, 1538-1548, doi:10.1128/JB.00010-15 (2015).

18 Tsai, H. H., Dwarakanath, A. D., Hart, C. A., Milton, J. D. \& Rhodes, J. M. Increased faecal mucin sulphatase activity in ulcerative colitis: a potential target for treatment. Gut 36, 570-576, doi:10.1136/gut.36.4.570 (1995).

19 Alipour, M. et al. Mucosal Barrier Depletion and Loss of Bacterial Diversity are Primary Abnormalities in Paediatric Ulcerative Colitis. J Crohns Colitis 10, 462-471, doi:10.1093/ecco-jcc/jjv223 (2016).

20 Kang, S. S. et al. An antibiotic-responsive mouse model of fulminant ulcerative colitis. PLoS Med 5, e41, doi:10.1371/journal.pmed.0050041 (2008).

21 Hickey, C. A. et al. Colitogenic Bacteroides thetaiotaomicron Antigens Access Host Immune Cells in a Sulfatase-Dependent Manner via Outer Membrane Vesicles. Cell host \& microbe 17, 672-680, doi:10.1016/j.chom.2015.04.002 (2015). 
22 Barbeyron, T. et al. Matching the Diversity of Sulfated Biomolecules: Creation of a Classification Database for Sulfatases Reflecting Their Substrate Specificity. PloS one 11, e0164846, doi:10.1371/journal.pone.0164846 (2016).

Hanson, S. R., Best, M. D. \& Wong, C. H. Sulfatases: structure, mechanism, biological activity, inhibition, and synthetic utility. Angewandte Chemie 43, 5736-5763, doi:10.1002/anie.200300632 (2004).

Hettle, A. G. et al. The Molecular Basis of Polysaccharide Sulfatase Activity and a Nomenclature for Catalytic Subsites in this Class of Enzyme. Structure 26, 747-758 e744, doi:10.1016/j.str.2018.03.012 (2018).

928

25 Lapebie, P., Lombard, V., Drula, E., Terrapon, N. \& Henrissat, B. Bacteroidetes use

929

930

931

932 thousands of enzyme combinations to break down glycans. Nature communications 10, 2043, doi:10.1038/s41467-019-10068-5 (2019).

933

934

Pudlo, N. A. et al. Extensive transfer of genes for edible seaweed digestion from marine to human gut bacteria. bioRxiv, 2020.2006.2009.142968, doi:10.1101/2020.06.09.142968 (2020).

27 Roche, P. et al. Molecular basis of symbiotic host specificity in Rhizobium meliloti: nodH and nodPQ genes encode the sulfation of lipo-oligosaccharide signals. Cell 67, 1131-1143, doi:10.1016/0092-8674(91)90290-f (1991).

937

28 Byrne, D. P. et al. cAMP-dependent protein kinase (PKA) complexes probed by complementary differential scanning fluorimetry and ion mobility-mass spectrometry. The Biochemical journal 473, 3159-3175, doi:10.1042/BCJ20160648 (2016).

940

Das, T. M., Rao, C. P. \& Kolehmainen, E. Synthesis and characterisation of N-glycosyl amines from the reaction between 4,6-O-benzylidene-D-glucopyranose and substituted aromatic amines and also between 2-(o-aminophenyl)benzimidazole and pentoses or hexoses. Carbohydr Res 334, 261-269, doi:10.1016/s00086215(01)00202-6 (2001).

946

947 Kabsch, W. Xds. Acta crystallographica. Section D, Biological crystallography 66, 125132, doi:10.1107/S0907444909047337 (2010).

31 Evans, P. Scaling and assessment of data quality. Acta crystallographica. Section D, Biological crystallography 62, 72-82, doi:10.1107/S0907444905036693 (2006).

Evans, P. R. An introduction to data reduction: space-group determination, scaling and

950

951

952 intensity statistics. Acta crystallographica. Section D, Biological crystallography 67, 282-292, doi:10.1107/S090744491003982X (2011).

33 Long, F., Vagin, A. A., Young, P. \& Murshudov, G. N. BALBES: a molecular-replacement pipeline. Acta crystallographica. Section D, Biological crystallography 64, 125-132, doi:10.1107/S0907444907050172 (2008).

956

957

34 McCoy, A. J. Solving structures of protein complexes by molecular replacement with Phaser. Acta crystallographica. Section D, Biological crystallography 63, 32-41, doi:10.1107/S0907444906045975 (2007).

958

35 Emsley, P., Lohkamp, B., Scott, W. G. \& Cowtan, K. Features and development of Coot.

959

960 Acta Crystallogr D Biol Crystallogr 66, 486-501, doi:10.1107/S0907444910007493 (2010).

961 structures. Acta crystallographica. Section D, Biological crystallography 67, 355-367, doi:10.1107/S0907444911001314 (2011). 
37 Lebedev, A. A. et al. JLigand: a graphical tool for the CCP4 template-restraint library. Acta crystallographica. Section D, Biological crystallography

68, 431-440, doi:10.1107/S090744491200251X (2012).

Chen, V. B. et al. MolProbity: all-atom structure validation for macromolecular crystallography. Acta crystallographica. Section D, Biological crystallography 66, 1221, doi:10.1107/S0907444909042073 (2010).

39 Potterton, L. et al. CCP4i2: the new graphical user interface to the CCP4 program suite. Acta Crystallogr D Struct Biol 74, 68-84, doi:10.1107/S2059798317016035 (2018).

972

40 Collaborative Computational Project, N. The CCP4 suite: programs for protein crystallography. Acta crystallographica. Section D, Biological crystallography 50, 760763, doi:10.1107/S0907444994003112 (1994).

41 Katoh, K., Misawa, K., Kuma, K. \& Miyata, T. MAFFT: a novel method for rapid multiple sequence alignment based on fast Fourier transform. Nucleic Acids Res 30, 3059-3066, doi:10.1093/nar/gkf436 (2002).

979

42 Clamp, M., Cuff, J., Searle, S. M. \& Barton, G. J. The Jalview Java alignment editor.

980 Bioinformatics 20, 426-427, doi:10.1093/bioinformatics/btg430 (2004).

981

43 Stamatakis, A. RAxML version 8: a tool for phylogenetic analysis and post-analysis of

982 large phylogenies. Bioinformatics 30, 1312-1313, doi:10.1093/bioinformatics/btu033 (2014).

983

44 Felsenstein, J. Evolutionary trees from DNA sequences: a maximum likelihood

984 approach. J Mol Evol 17, 368-376, doi:10.1007/BF01734359 (1981).

985

45 Le, S. Q. \& Gascuel, O. An improved general amino acid replacement matrix. Mol Biol

986

987 Evol 25, 1307-1320, doi:10.1093/molbev/msn067 (2008).

988

46 Felsenstein, J. Confidence Limits on Phylogenies: An Approach Using the Bootstrap.

989 Evolution 39, 783-791, doi:10.1111/j.1558-5646.1985.tb00420.x (1985).

990

47 Varki, A. et al. Symbol Nomenclature for Graphical Representations of Glycans. 991 Glycobiology 25, 1323-1324, doi:10.1093/glycob/cwv091 (2015). 

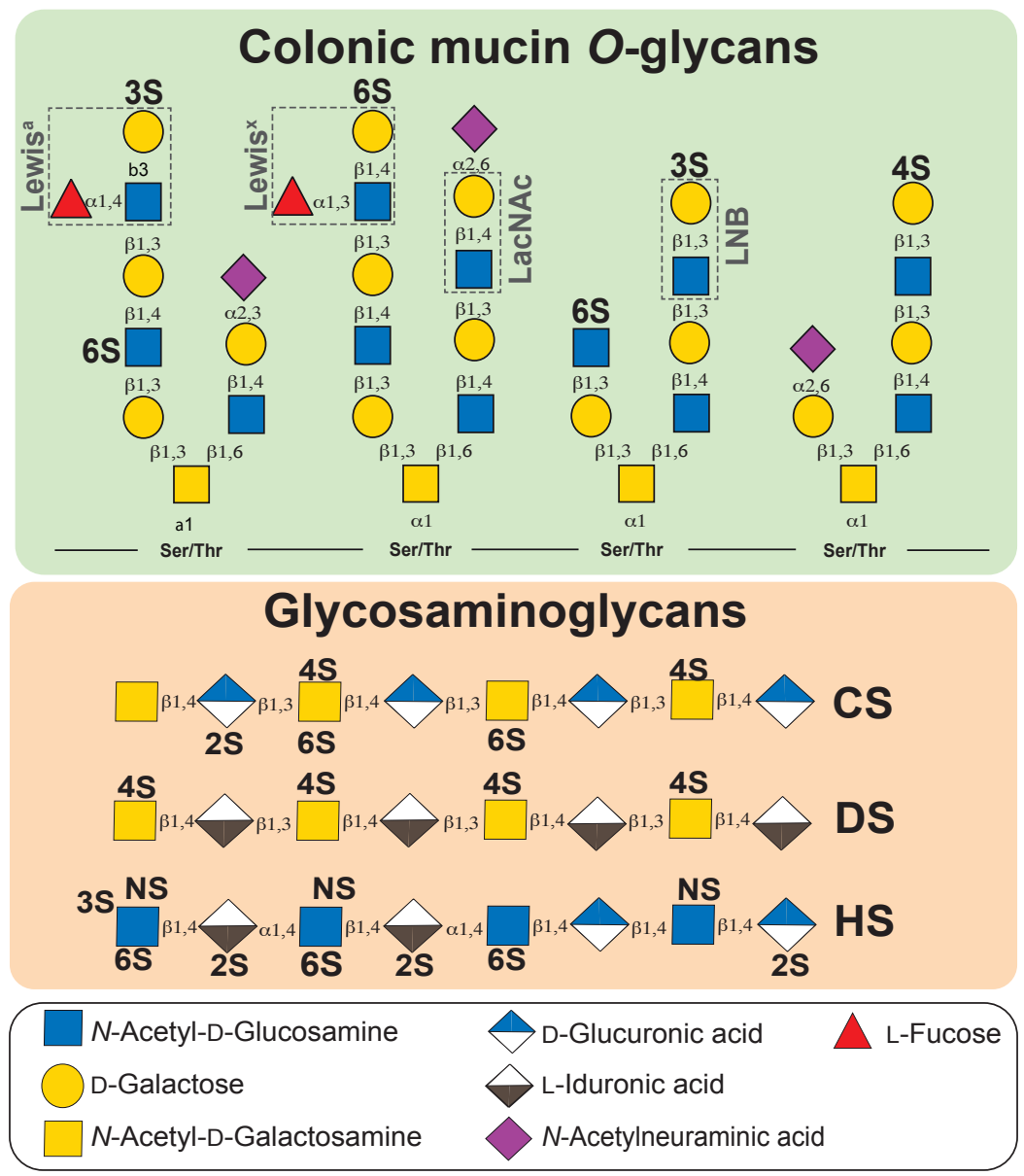

Figure 1. Examples of sulfated host carbohydrates found in the colon

Schematic representation of sulfated host glycans found in mucin O-glycans (green box) and glycosaminoglycans which are integral to the extracellular matrix and glycocalyx (peach box), representing a constant, host derived, nutrient source for the colonic microbiota. Sugars are shown according to the Symbol Nomenclature for Glycan system ${ }^{47}$. 

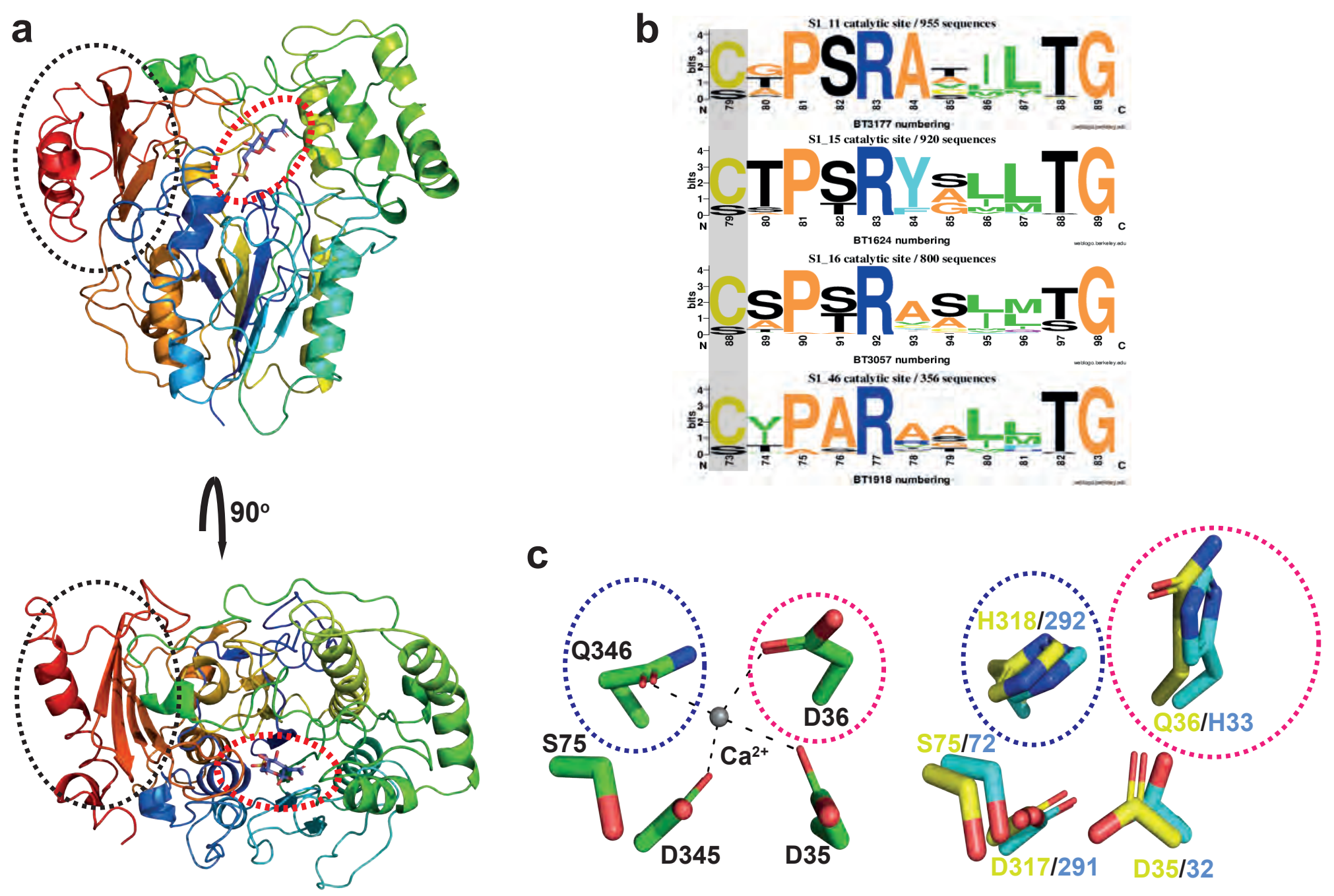

Figure 2. Conserved features of $\mathbf{S 1}$ carbohydrate sulfatases.

a, Example of the overall fold common to all S1 sulfatases, Cartoon representation of BT31776S-GlcNAc colour ramped from blue ( $\alpha / \beta / \alpha \mathrm{N}$-terminal domain) to red ( $\beta$ - sheet $\mathrm{C}$-terminal domain). The black dashed circle indicates the C-terminal sub-domain and the red dashed circle the active site location on the core $\alpha / \beta / \alpha$ domain. $b$, Conservation of the consensus active site sequence for S1 11, S1_15, S1 16 and S1 46. The position of formylglycine installation is highlighted with a grey box c, Examples of the calcium binding site: left side (green), representation of BT31776S GlcNAc calcium site as an example of the typical site observed in most sulfatase structures described to date; right side, display of alternate sites observed in BT19183S-GIcNAc (yellow) and BT15962S- $\Delta 4$,5UA (cyan). The variable areas are highlighted in blue and red dashed circles. 
a
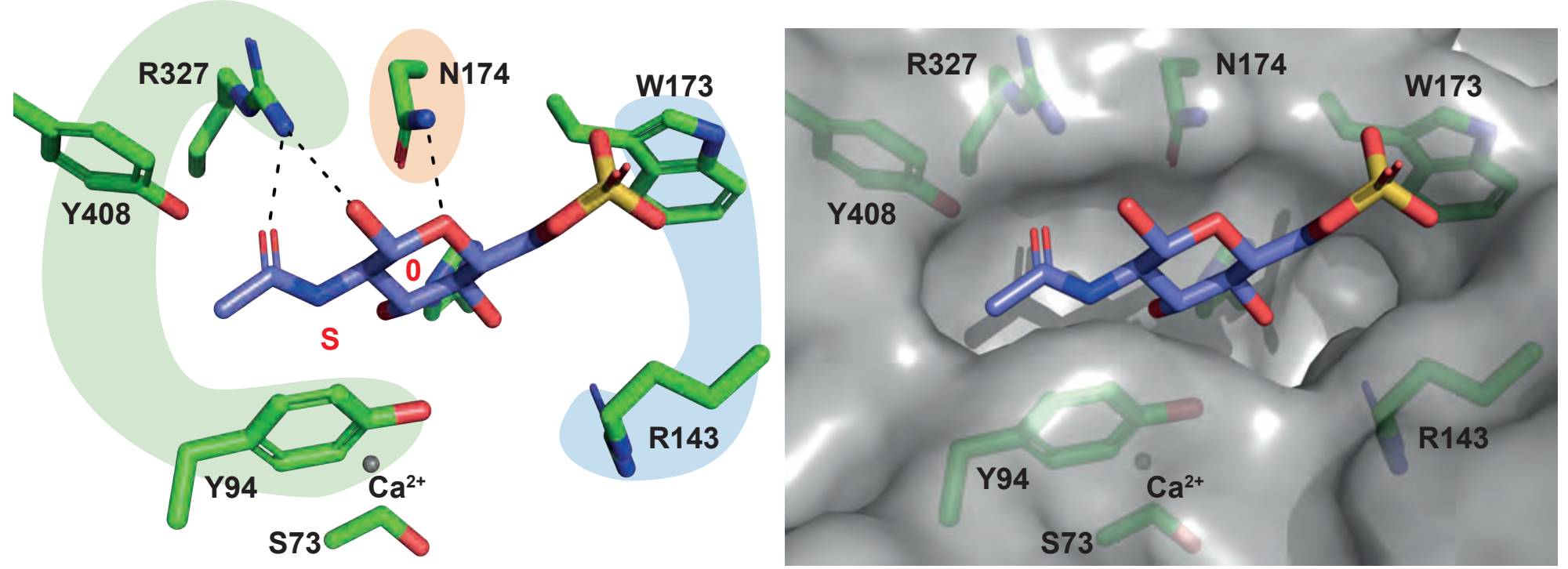

b

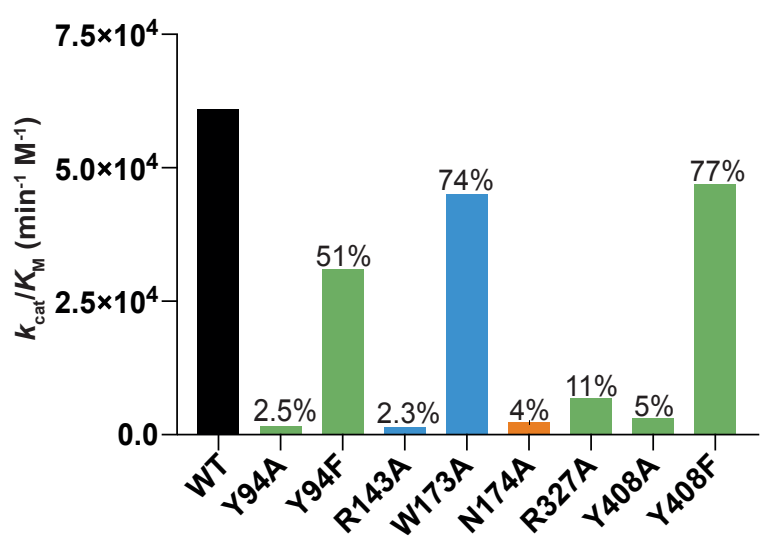

C

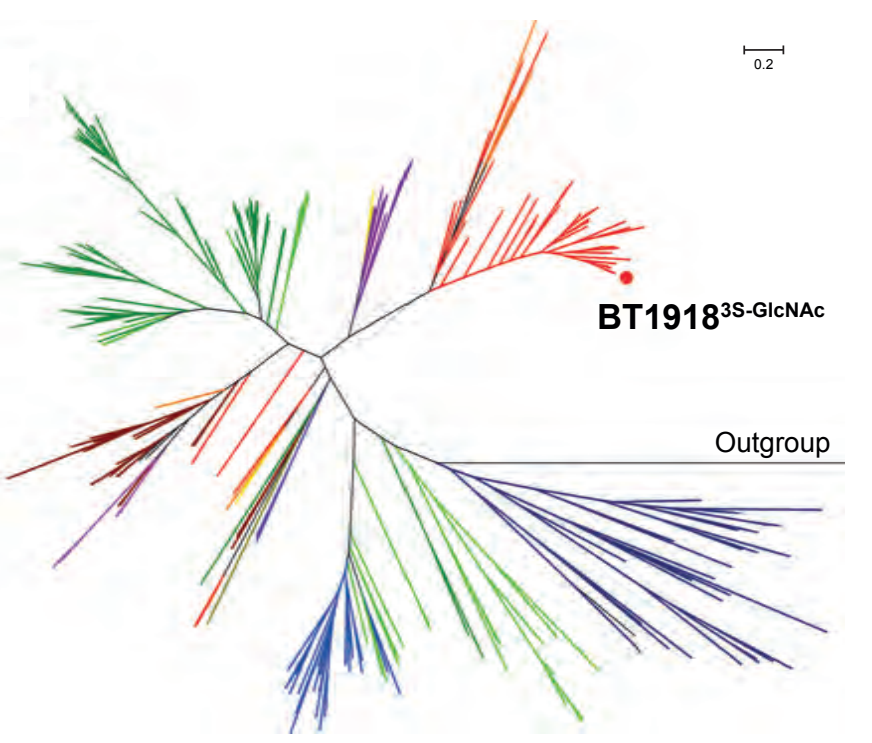

Figure 3. Structural details of the carbohydrate binding region of BT19183S-GICNAC

a, Stick representation of the structural data showing the carbohydrate binding interactions of the 0 subsite of BT1918 ${ }^{3 \mathrm{~S}-G l c N A c}$ (left panel) and surface representation of the structural data for BT19183S-GlcNAc showing the 0 subsite pocket (right panel). Highlighted in green are the residues interacting with the $\mathrm{N}$-acetyl, in blue are the sulfate flanking residues, and orange indicates sugar ring only interactions. $\mathbf{b}$, Catalytic effects of alanine scanning on BT1918 ${ }^{3 \mathrm{~S}-\mathrm{GICNAC}}$. Percentages above the bar indicate relative activity to wildtype (WT). c, Radial version of the phylogenetic tree of representative sulfatases from subfamily S1_46. For clarity all labels and sequence accession codes have been omitted. The annotations next to the colour code concern the presence or absence of conservation of the residues crucial in substrate recognition by BT19183S-GlcNAc (acc-code Q8A6G6) and in the following order: Y94, N174, R327 and Y408. The residues are coloured as following: black means an equivalent residue is present; a grey and bold letter at any position means that the corresponding residue is replaced by that amino acid; a grey, bold and italic letter at any position means that the equivalent position is replaced by any type of amino acid; a bold grey letter followed by one-letter codes in parentheses indicates that the equivalent position can be substituted by any of those amino acids; the dash at the Y408equivalent position indicates that no equivalent amino acid can be deduced from the multiple alignment. Branches having the same colour have the corresponding pattern in common. The red filled circle designates the sequence of the S1_46 sulfatase from B. thetaiotaomicron (See Figure S8 for full tree). 
a

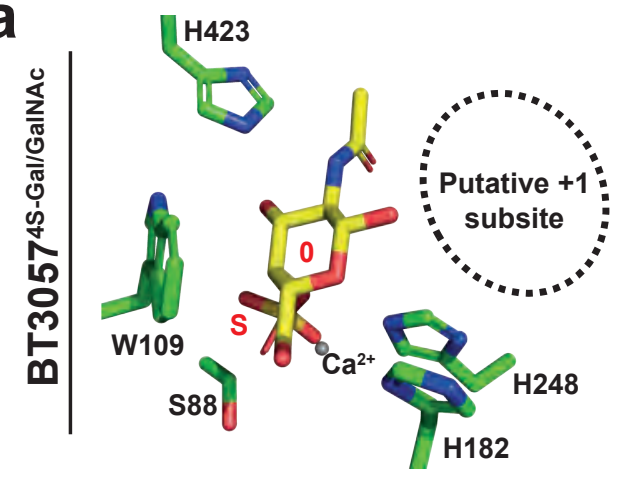

b

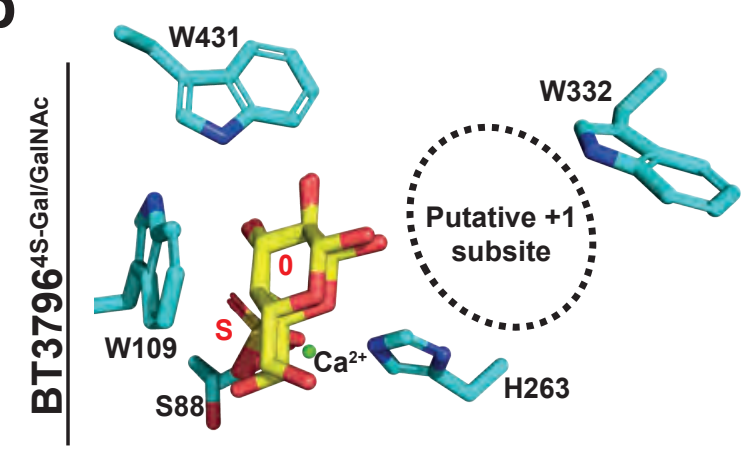

C
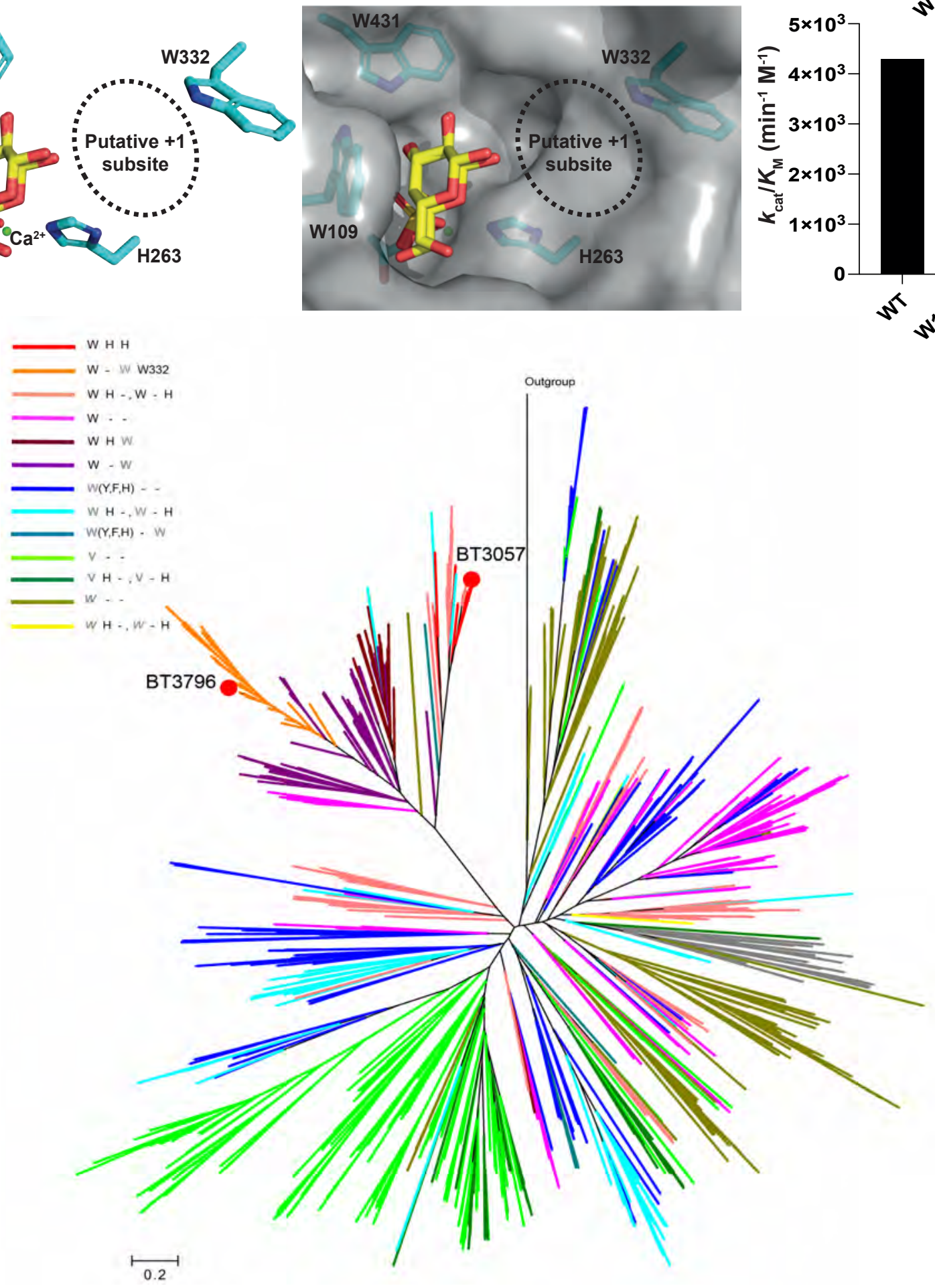
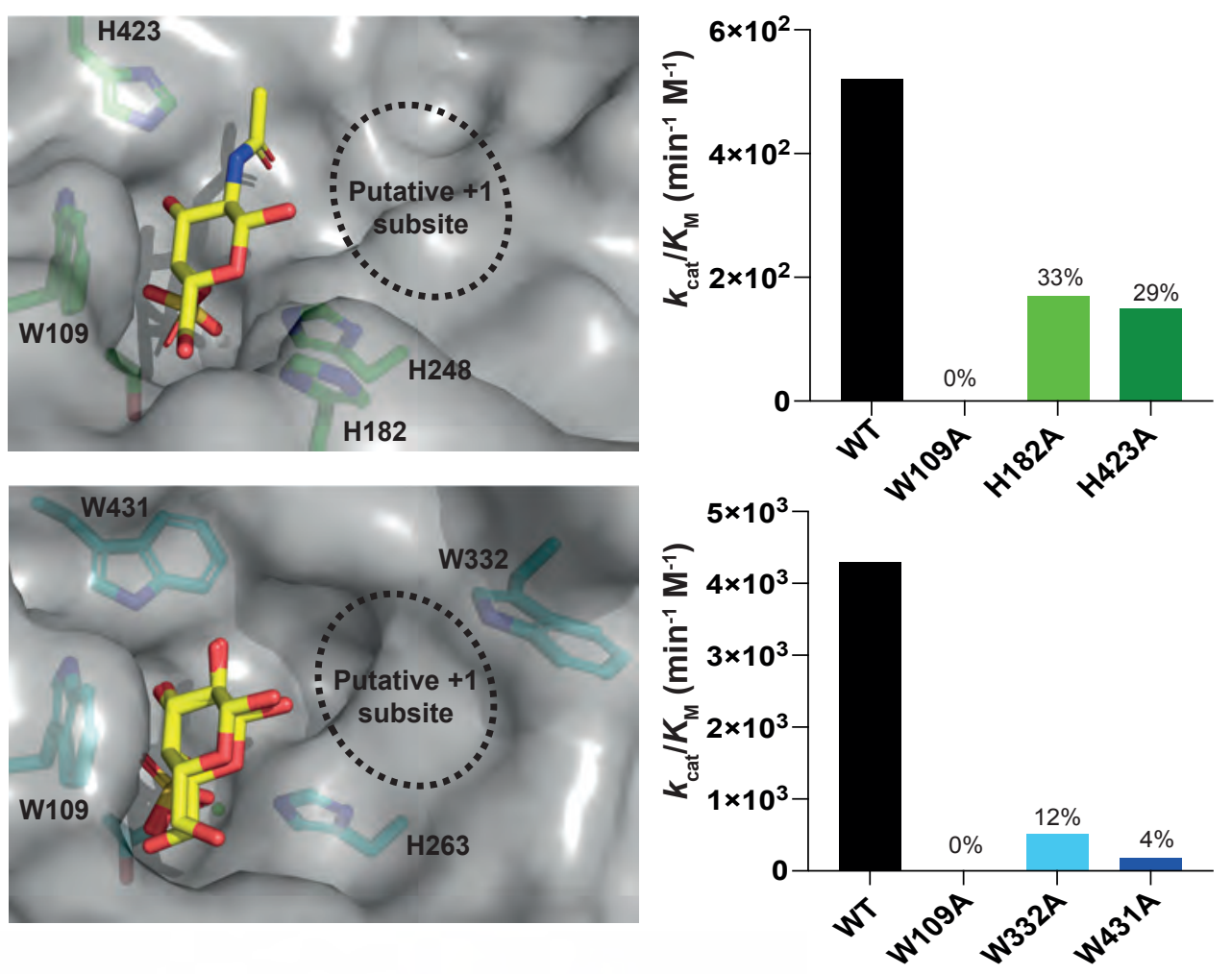
Figure 4. Structural details of the carbohydrate binding region of BT30574S Gal/GalNAc and BT37964S Gal/ GalNAc

Stick (left) and surface (middle) representation showing the carbohydrate binding interactions of the 0 subsite of S1_16 sulfatases: a, BT30574S-Gal/GalNAc and b, BT37964S-Gal/GalNAc. The respective right panel show the effects of alanine scanning on these sulfatase activities with the percentages above the bar indicating the relative activity to wildtype (WT). c, Radial phylogenetic tree of representative sulfatases from subfamily S1_16. For clarity all labels and sequence accession codes have been omitted. The annotations next to the colour code concern the presence or absence of conservation of the critical residues in substrate recognition by BT30574S-Gal/GalNAc (acc-Code Q8A397) in the order: W109, H182 and H423. Sequences coded by orange branches contain an additional W332 present in BT37964S-Gal/GalNAc (acc-code Q8A171) but absent in other sequences. For simplification the residue numbers have been omitted, except for W332. The residues are coloured as following: black means an equivalent amino acid is present; a grey and bold letter at any position means that the corresponding residue is replaced by that amino acid; a grey and italic letter at any position means that the equivalent position is replaced by any type of amino acid; a bold grey letter followed by one-letter codes in parentheses indicates that the equivalent position can be substituted by any of those amino acids; the dash at the $\mathrm{H}$-equivalent position indicates that no equivalent amino acid can be deduced from the multiple alignment. When two patterns are indicated separated by a comma (i.e. W - H, W H -) both have been attributed the same colour code. Branches having the same colour have the corresponding pattern in common. Red filled circles designate sequences of S1_16 sulfatases from B. thetaiotaomicron (See Figure S9 for full tree). 
a
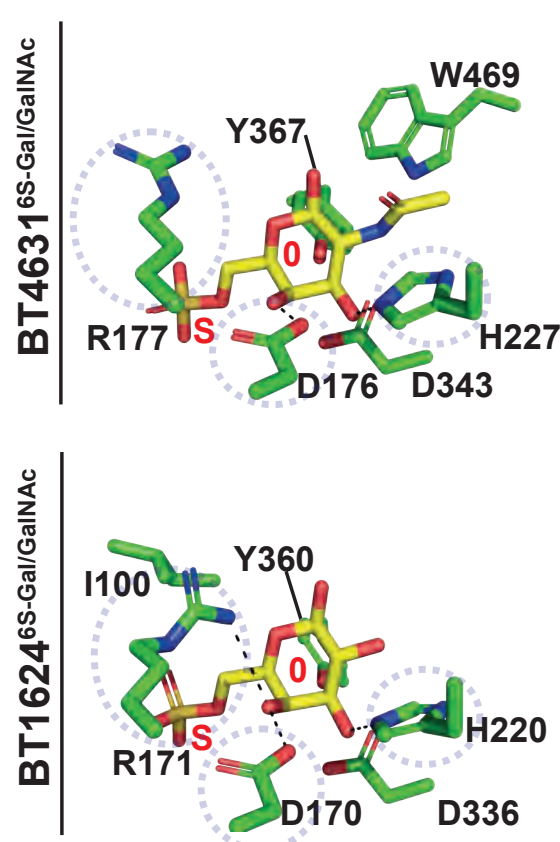



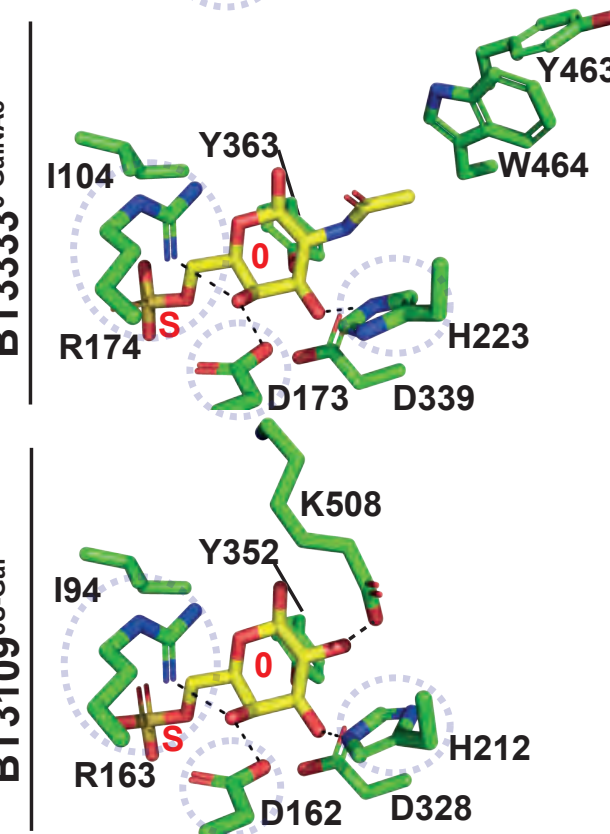

b
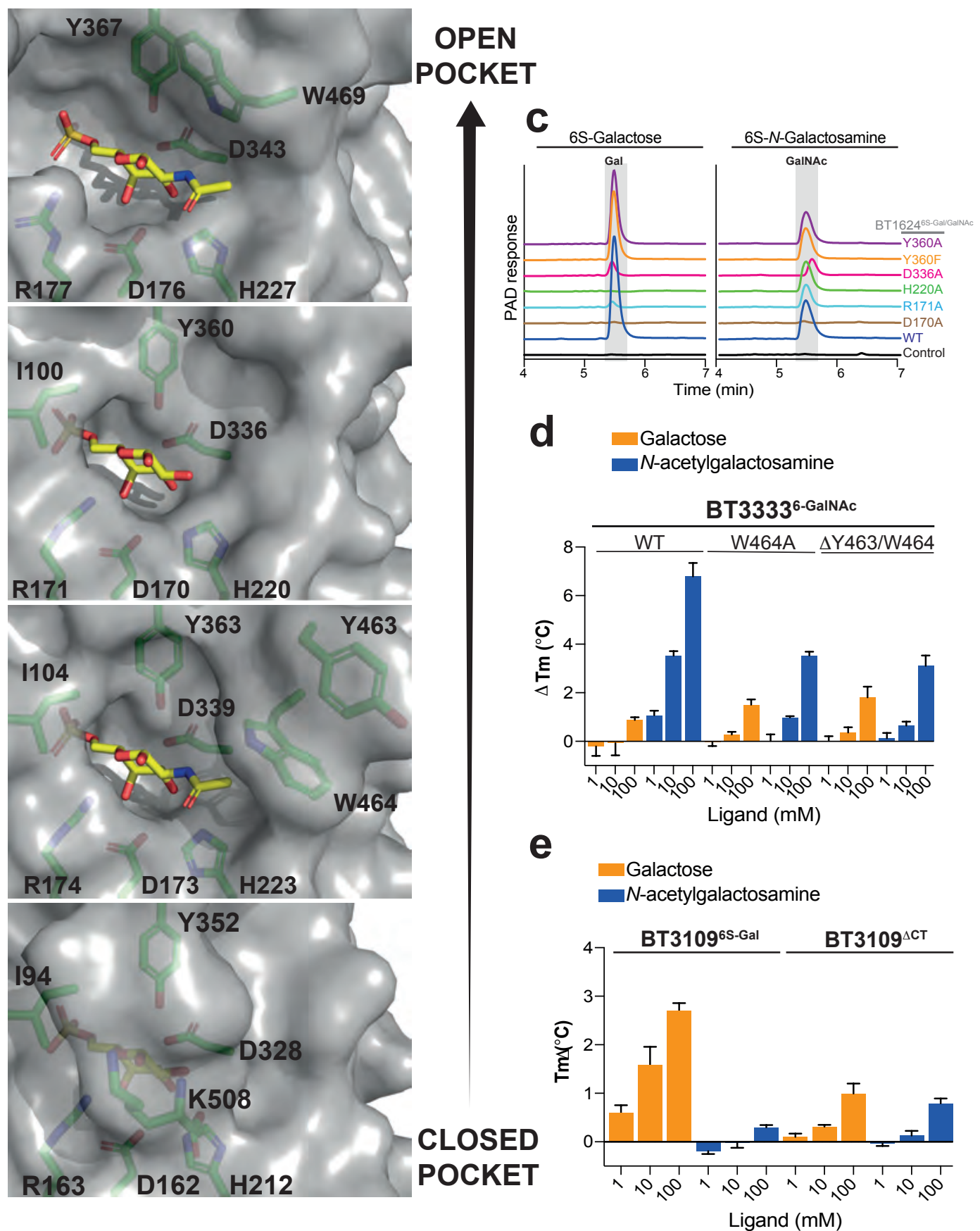

Figure 5. Structural variations of the carbohydrate binding region of the S1_15 family

$\mathbf{a}$, Stick and $\mathbf{b}$, Surface representation showing the carbohydrate binding interactions of the 0 subsite of BT46316SGall'GalNAc, BT16246S-Gal/GalNAc, BT33336S-GalNAc, and BT31096S-Gal (from top to bottom). c, High pressure anion exchange chromatography '(HPAEC) of wildtype BT1624 ${ }^{6 S-G a l / G a l N A c}$ wildtype (WT) and its mutants. The produced product is highlighted by a grey box. HPAEC reactions utilised $6 \mathrm{mM}$ substrate and $5 \mathrm{M}$ enzyme, with $3 \mathrm{mM}$ HEPES, $45 \mathrm{mM} \mathrm{NaCl}$, and $5 \mathrm{mM} \mathrm{CaCl} 2$ over a $48 \mathrm{~h}$ period at $37^{\circ} \mathrm{C}$. d, DSF analysis of the effect of mutating the GalNAc specificity features of BT33336S-GalNAc. e, DSF analysis of the effect of mutating the C-terminal extension (VEEEPLK) which drives specificity towards Gal in BT31096S-Gal. 100 mM BTP pH 7.0 with 150 mM NaCl was used in all DSF experiments. Error bars represent s.e.m.. 
a

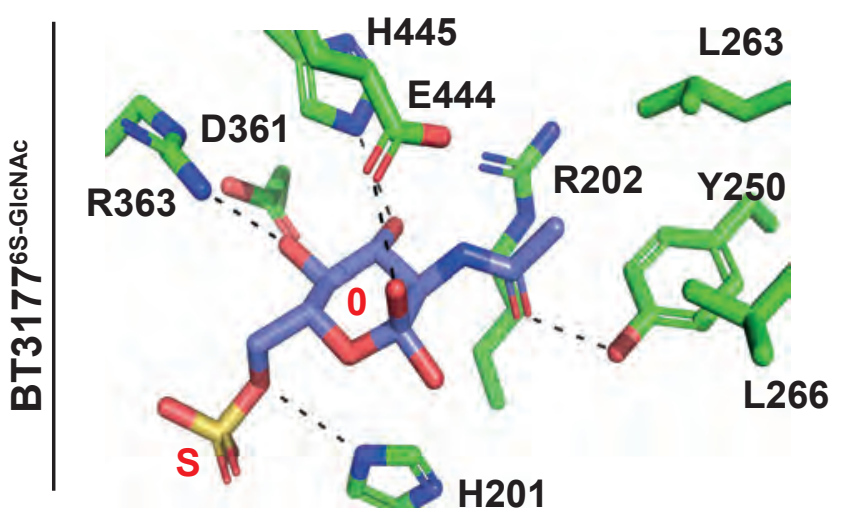

b

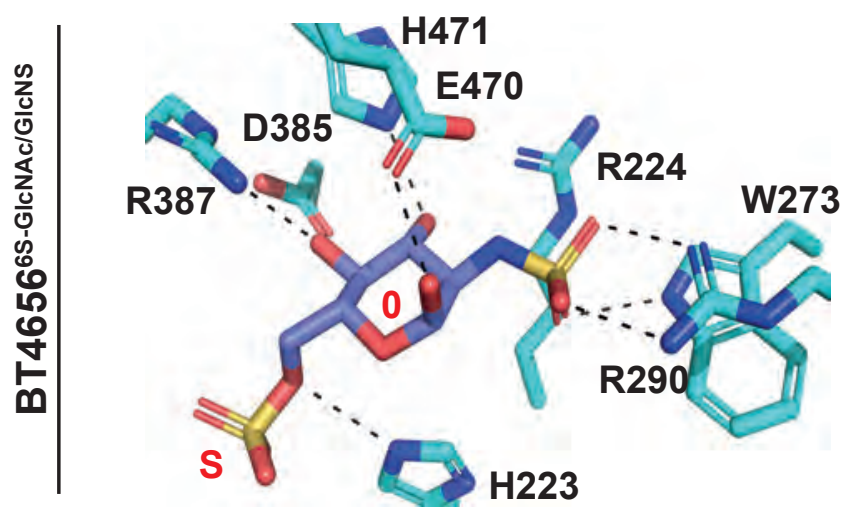

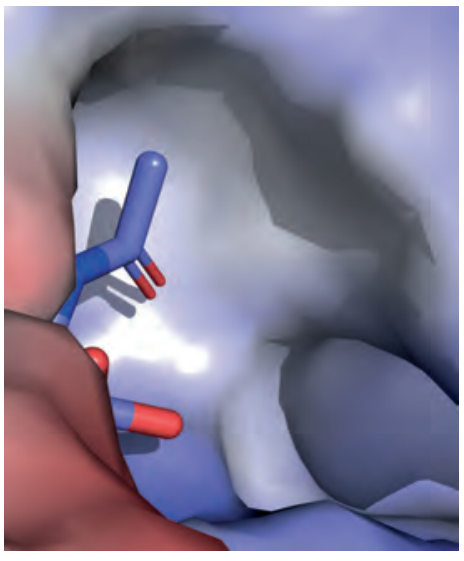

C
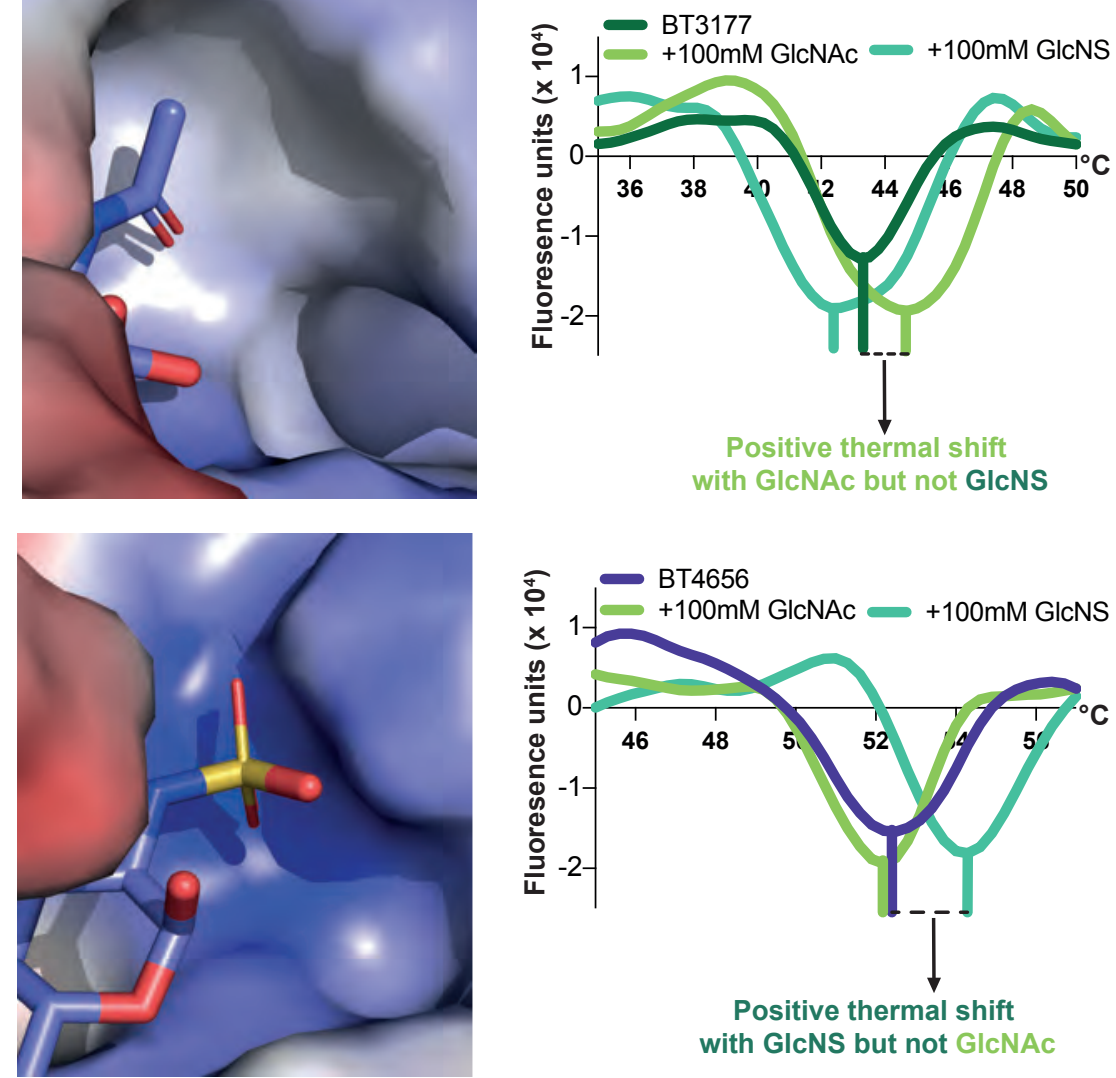

Figure 6. Structural variations of the carbohydrate binding region of the S1_11 family

a, Carbohydrate interactions of BT3177 ${ }^{6 S-G I C N A c}$. b, Carbohydrate interactions of BT4656 6 S-GIINAc/GICNS. In both panels the left is a stick representation of the carbohydrate interactions at S0, middle is a surface representation showing the local surface charge. c, DSF data showing the second derivative of the thermal

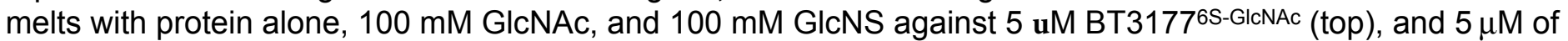
BT4656 6S-GICNAC/GICNS (bottom). 


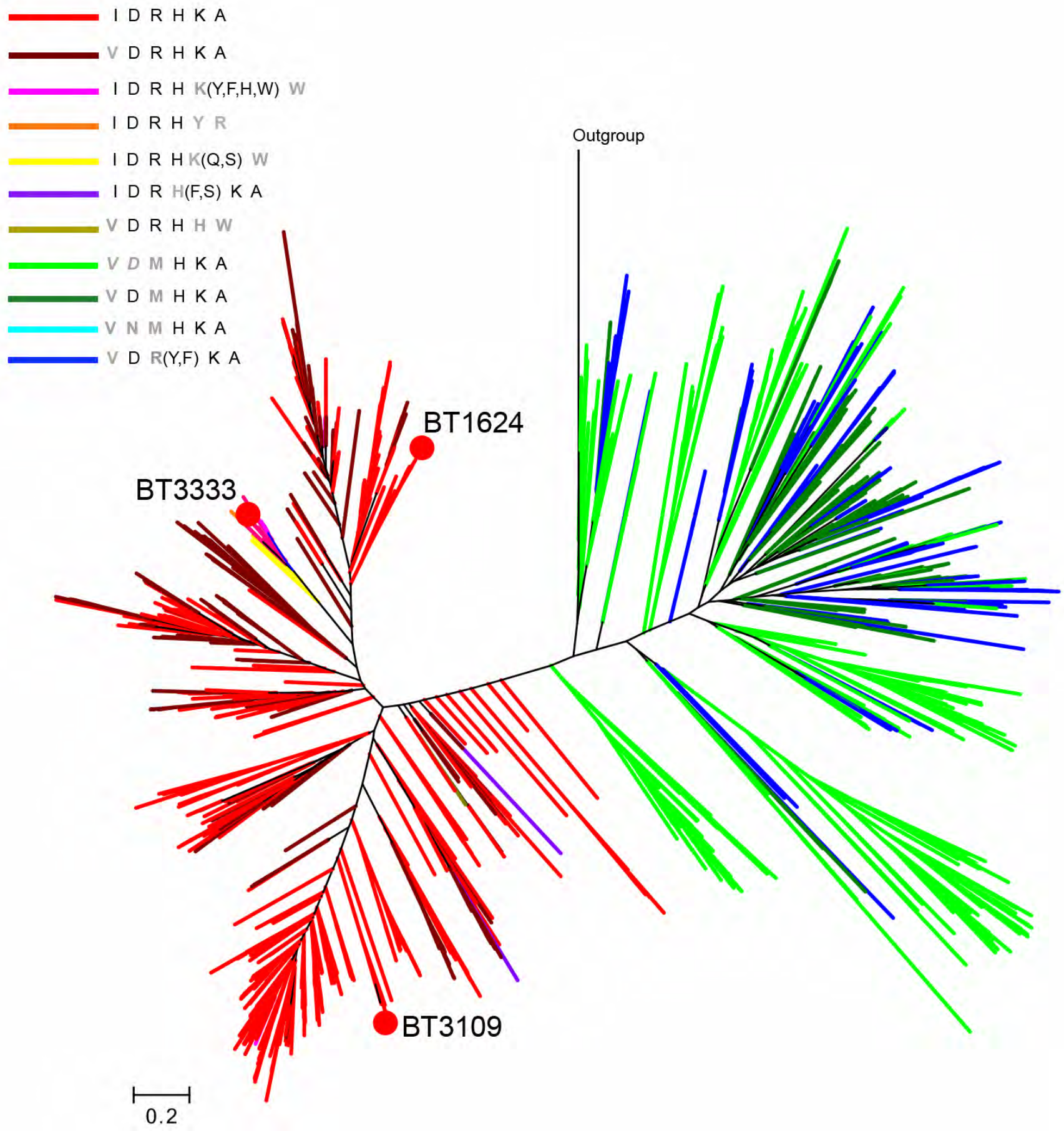

Extended data 1. Radial version of the phylogenetic tree of representative sulfatases from subfamily S1_15.

For clarity all labels and sequence accession codes have been omitted. The annotations next to the colour code concern the presence or absence of conservation of the indicated residues and in this order: $\mid 100$, D170, R171, H220, K461 and A462. These residues are crucial in substrate recognition by BT16246S-Gal/ GalNAc (acc-code Q8A7A1). For simplification the residue numbers have been omitted. For example, an I in black means an equivalent isoleucine is present; a grey and bold letter at any position means that the corresponding residue is replaced by that amino acid; a grey and italic letter at any position means that the equivalent position can be replaced by any type of amino acid; a bold grey letter followed by one-letter codes in parentheses indicates that the equivalent position is substituted by any of those amino acids. Branches having the same colour have the corresponding pattern in common. Red filled circles designate sequences of S1_15 sulfatases from B. thetaiotaomicron (See Figure S10 for full tree). 


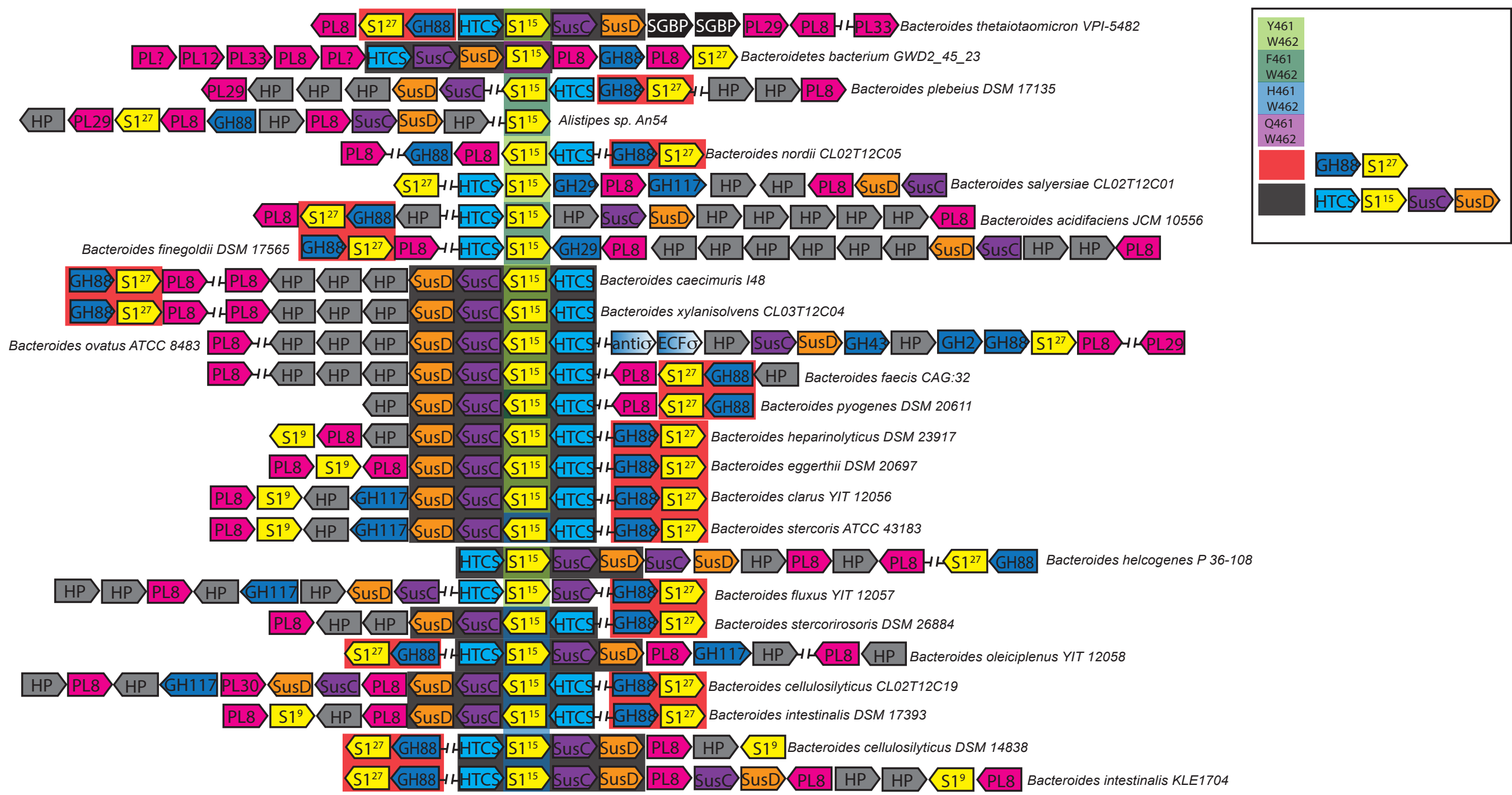

Extended data 2. Analysis of S1_15 enzymes with PULs targeting chondroitin sulfate.

PULs targeting chondroitin sulfate aligned by orthologues of BT33336S-GalNAc. Light green background shows orthologues with Y463/W464, a dark green background highlights orthologues with F463/W464, a light blue back ground highlight orthologues with H463/W464, and a purple background highlight orthologues with Q463/W464. The numbering used corresponds to the sequence of BT33336S-GalNAc. A red background highlights the presence of GH88 and S1_27 (an endo 4S-chrondroitin sulfatase) which appear to be discrete genetic block not always physically localised to the PUL. A black background highlights a core block observed in CS PULs containing BT3333 ${ }^{6 S-G a l N A c}$ orthologues.

\section{Extended data 2}




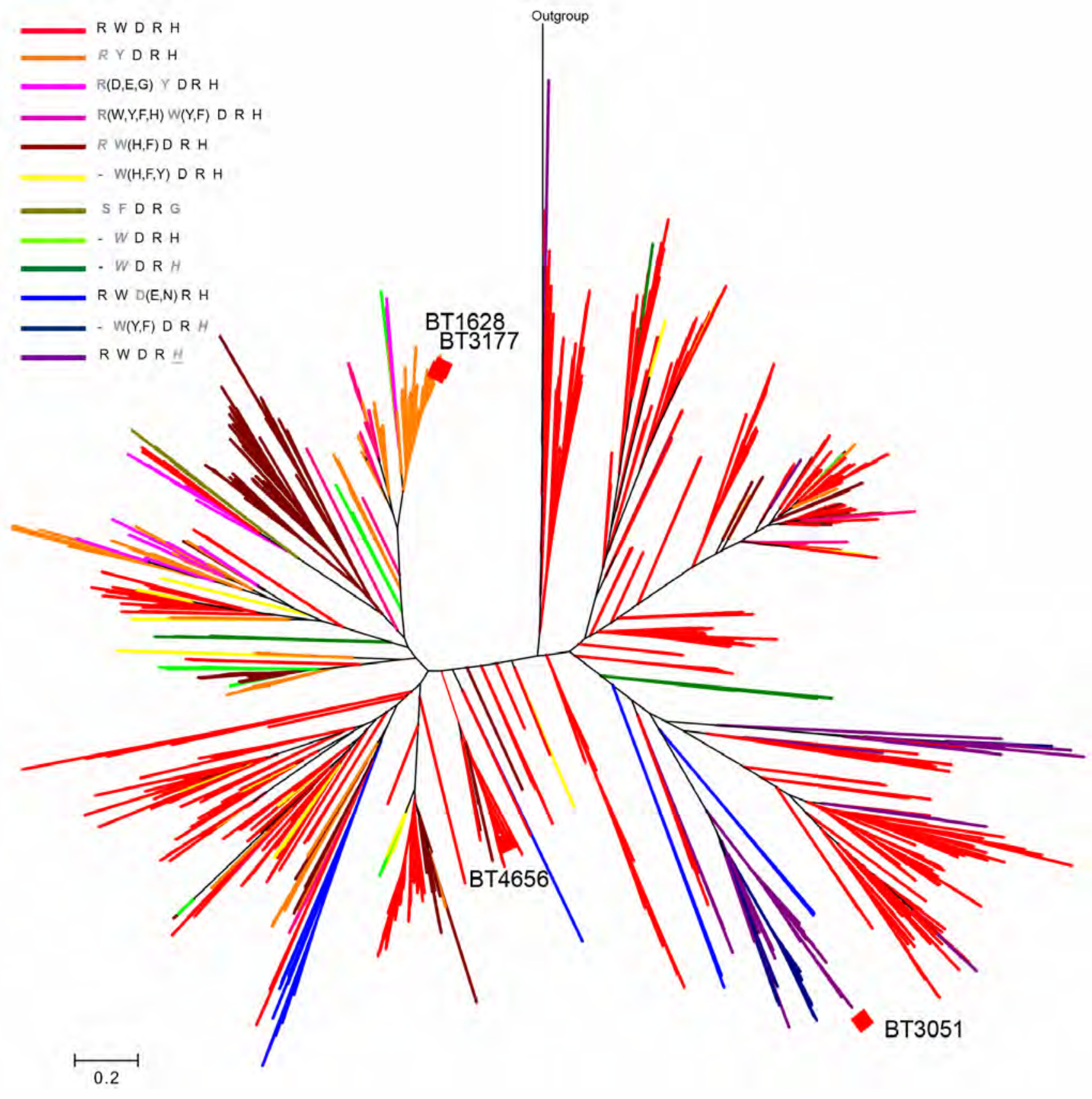

\section{Extended data 3. Radial version of the phylogenetic tree of representative sulfatases from subfamily} S1_11

For clarity all labels and sequence accession codes have been omitted. The annotations next to the colour code concern the presence or absence of conservation of the indicated residues and in this order: R290, W273, D385, R387 and H471. These residues are crucial in substrate recognition by BT4656 6S-GlcNAc/GlcNS (acc-code Q89YS5). For simplification the residue numbers have been omitted. For example, a $\mathrm{R}$ in black means an equivalent arginine is present; a grey and bold letter at this position means that the corresponding residue is replaced by that amino acid; the grey and italic $R$ at this position means that the R-equivalent position is replaced by any type of amino acid; a bold grey $R$ followed by one-letter codes in parentheses indicates that the R-equivalent position can be substituted by any of those amino acids; the dash at the R-equivalent position indicates that no equivalent amino acid can be deduced from the multiple alignment. Branches having the same colour have the corresponding pattern in common. Red filled diamonds designate sequences of S1_11

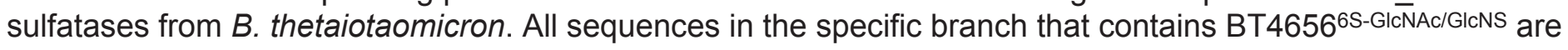
found within a conserved heparin PUL (See Figure S11 for full tree). 
PL15) SusC SusD HTCS HP PL12 S1 S111 S1 S1 HP ROK PL15 GH80 Bacteroidetes bacterium GWD2_45_23

HTC) PL12 HP SusC SusD SuSD PL8 DUF GH95 GH95 GH88 PL12 PL12 S111 DUF ROK DUF PL15 Bacteroides reticulotermitis JCM 10512

PL15 ROK HP S111 PL12 GH88 PL8 SusD SUSC SGBP PL12 HP DUF DUF HTCS Bacteroides faecis MAJ27

PL15 MFS ROK HP S111 PL12 GH88 SUSD SUSC SGBP PL12 HTCS Bacteroides nordii CL02T12C05

S11 PL12 GH88 SUSD SUSC SGBP PL12 HP HTCS Bacteroides xylanisolvens SD CC 1b

PL15 MFS ROK HP S111 PL12 GH88 SUSD SUSC SGBP PL12 HTCS-1 PL13 Bacteroides caecimuris 148

PL15 MFS ROK HP S111 PL12 GH88 SUSD SUSC SGBP PL12 HTCS-11 PL13 Bacteroides ovatus ATCC 8483

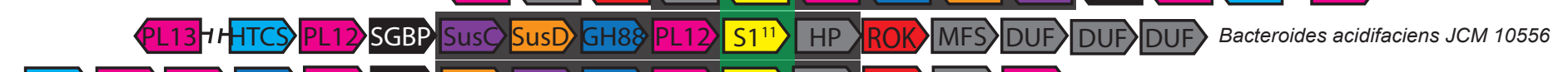

HTC5 PL12 PL12 GH95) PL12 SGBP SuSC SusD GH8\% PL12 S111) HP ROK MFS PL15 Bacteroides finegoldii DSM 17565

MFS ROK HP S111 PL12 GH88 SUSD SUSC SGBP PL12 HTCS-1 PL13 Bacteroides eggerthii DSM 20697

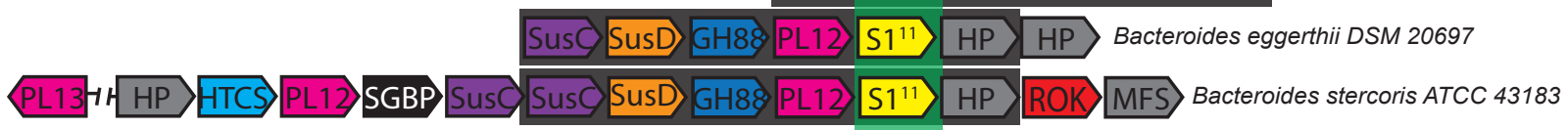

PL1311 1 ITCS PL12) SGBP SusC SusD GH80 PL12 S111 HP ROK MFS Bacteroides clarus YIT 12056

S18 PL37) HTCS SUS SUSD HP S17 S111 Bacteroides clarus YIT 12056

Sus SUSD HP S111 S18 Bacteroides pyogenes DSM 20611

Sus SuSD GH88 PL12 S111 HP ROK MFS PL15 S19 Bacteroides helcogenes P 36-108

PL12 GH8 PL15 Sus SusD S111 HP ROK MFS Prevotella bergensis DSM 17361

PL13 S18 HP HTCS EPI SUS SUSD GH80 PL12 S111 HP ROK MFS PL15 Bacteroides stercorirosoris DSM 26884

GH88 PL12 SGBPSUSD SUSC PL15 PL15 HTCS S111 Bacteroides oleiciplenus YIT 12058

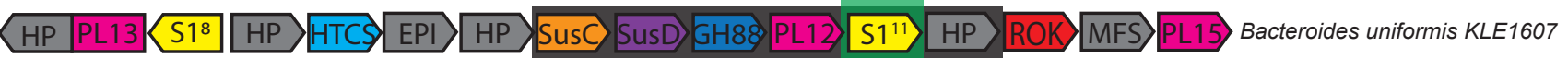

S19 PL15 MFS ROK HP S111 PL12 GH88 SUSD SUSC SGBP PL12 EPI HTCS PL37 S18 PL13) Bacteroides intestinalis DSM 17393

HP S11 PL12 GH88 SUSD SUSC EPI HTCS PL37 S18 PL13 Bacteroides cellulosilyticus DSM 14838

HTCS PL15 ROK HP S111 S19 PL12 PL12 PL13 Alistipes timonensis JC136

HTC5 HP PL12 PSS S111 HP ROK MFS PSS PL13 HP PSS SusCSUSD PL15 Coprobacter secundus 177 R290Q

Pedobacter heparinus DSM 2366 HTCS S19 S18 S111 SUSD SUSC GH80 S169 HTCS PL15 HP PL13 S19 HP PL8 GH88 HP GH29 GH29 SUSD SUSCGH88 HTCS PL15 S19 S18 S11 PL12 SUSD SUSC GH88 PL12 Pseudopedobacter saltans DSM 12145

Extended data 4. Analysis of S1_11 enzymes with PULs targeting chondroitin sulfate.

PULs targeting heparan sulfate (HS) aligned by orthologues of BT46566S-GICNAc/GICNS. Orthologues of BT46566S-GICNAc/GICNS with W273/R290 and W273/

Q290 are highlighted with green and blue background, respectively. A black background highlights a core block observed in HS PULs containing BT46566S-GICNAC/GICNS orthologues.

\section{Extended data 4}

